\title{
Value- and Ambiguity-Based Approach for Solving Intuitionistic Fuzzy Transportation Problem with Total Quantity Discounts and Incremental Quantity Discounts
}

\author{
C. Veeramani ${ }^{1},{ }^{1}$ M. Joseph Robinson, ${ }^{2}$ and S. Vasanthi ${ }^{3}$ \\ ${ }^{1}$ Department of Applied Science (Mathematics), PSG College of Technology, Tamil Nadu 641004, Coimbatore, India \\ ${ }^{2}$ Department of Mathematics, Gojan School of Business and Technology, Chennai, Tamil Nadu 600052, India \\ ${ }^{3}$ Department of Mathematics, Rajalakshmi Engineering College, Chennai, Tamil Nadu 600025, India \\ Correspondence should be addressed to C. Veeramani; cvm.amcs@psgtech.ac.in
}

Received 17 September 2020; Revised 28 October 2020; Accepted 2 November 2020; Published 30 December 2020

Academic Editor: S. A. Edalatpanah

Copyright $(2020$ C. Veeramani et al. This is an open access article distributed under the Creative Commons Attribution License, which permits unrestricted use, distribution, and reproduction in any medium, provided the original work is properly cited.

\begin{abstract}
The cost of goods per unit transported from the source to the destination is considered to be fixed regardless of the number of units transported. But, in reality, the cost is often not fixed. Quantity discount is often allowed for large shipments. Furthermore, the transportation cost and the price break quantities are not deterministic. In this study, we introduce the concept of Value- and Ambiguity-based approach for solving the intuitionistic fuzzy transportation problem with total quantity discounts and incremental quantity discounts. Here, the cost and quantity price breakpoints are represented by trapezoidal intuitionistic fuzzy numbers. The Values and Ambiguities are defined as the degree of acceptance and rejection for trapezoidal intuitionistic fuzzy numbers. The trapezoidal intuitionistic fuzzy transportation problem is converted to a parametric transportation problem based on their Value indices and Ambiguity indices. Then, for different Values of the parameter, the transformed problem is reduced to the linear programming problem. Then, the linear programming problem is solved by using the classical methods. The proposed method is demonstrated with a numerical example. In conclusion, the intuitionistic fuzzy transportation problem with total quantity discounts is compared with the intuitionistic fuzzy transportation problem with incremental quantity discounts.
\end{abstract}

\section{Introduction}

In conventional transportation problems, it is assumed that the decision-maker is certain about the exact values of the cost of transportation, availability, and demand for the product. In real-world applications, all these parameters of the transportation problem may not be known accurately due to uncontrollable factors. For example, suppose a product is transported to a destination for the first time, and no one knows the cost of transportation. So, there is uncertainty about the cost of transportation. When a new product is launched in the market, for the first time there is always uncertainty about the demand for that particular product. In everyday life, suppose a buyer asks whether the particular product is available or not, and the supplier replies yes, but when the supplier searches for that product, it may not be available at that time. Sometimes, the supplier himself does not know the availability of the product. To deal with such situations, the fuzzy set theory is used in the literature to solve traffic problems. The transportation problem is a delivery-type problem. The main goal of this study is to find how to transfer goods from different dispatch locations (also called origins) to different receiving points (also called targets) with minimal costs or largest profit. A quantity discount is an incentive offered to a buyer; i.e., a reduction in the cost of a unit of goods while purchasing large quantities of goods. A quantity discount is often offered by sellers to buyers to buy large quantities so that the seller can move more goods or items and the buyer can receive a favourable price for the goods. At the consumer level, a one-size discount may appear as buy one and get a discount or other perks such as buying two and getting one free.

Chandran and Perry [2] found that the cost per unit of transportation for a given sink from a particular supply source 
depends on the quantity shipped; hence, there is limited capacity for the number of price breakpoints delivered to customers. In 1990, Das [3] examined that it is often helpful to consider limitations, intervals, and the integration of decision variables for responding to many practical needs. Lee et al. [4] discussed the result and said that due to today's increasing competitive market and the ever-changing marketplace and inventory, problem-solving is becoming more complicated. The incorporation of heuristic methods had become a new trend in the past decade to address complexity. Acharya et al. [5] inspected the generalized transportation problem and found that the traffic cost for a unit product is assumed to be independent of the number of goods transported from the origin to the target. Mubarack Ahmed and Emmanuel [6] stated that it is assumed that the cost of goods for a unit shipped from a particular source to a particular destination is determined by the sum of the goods. George et al. [7] discussed the use of the transportation algorithm in calculating the cost of delivery using the Nigerian Bottling Company Plc Owerri Plant. Das et al. [19] discovered an effective method to solve a completely purged linear programming problem. Jana [9] discussed the generalized intuitionistic fuzzy operations and developed the application of intuitionistic fuzzy transportation problem.

Dinagar and Thiripurasundari [10] proposed a new method to find a fuzzy optimal solution for the fuzzy transportation problem. In this work, intuitionistic trapezoidal fuzzy numbers are used to represent transportation costs. The fuzzy optimal solution obtained in this study is the same as the fuzzy MODI method or the fuzzy Vogel approximation method. In 2017, Ebrahimnejad and Verdegay [8] used the accuracy function in order to convert the formulated IFTP to a deterministic LP problem. Furthermore, Edalatpanah and Shahabi [18] provided a new twophase solution method for solving fuzzy linear programming without using artificial variables. Kokila et al. [11] developed an efficient method for seeking an optimal solution to type-2 trapezoidal intuitionistic fuzzy fractional transportation problems. Anju [12] discussed the hexagonal intuitionistic fuzzy fractional transportation problems using ranking and Russell methods. The field of intuitionistic traffic problems is very important, especially in everyday life, and its solutions are also important. Lakshmi and Vinotha [13] expressed that the most important goal of the article is to present a decision process without limitations on the cost of emissions by the weight of transport and the transport of multipurpose problems. Mishra et al. [14] described that the intellectual problem of fuzzy transportation with interval Values is solved by Bharti and Singh's method. It represents the optimal interval Value of the intuitionistic fuzzy (IVIF) transportation cost to obtain multiple IVTIFNs. Edalatpanah et al. [17] proposed an expanded DEA model in the triangular intuitionistic fuzzy number environment with DIF inputs and TIFN outputs based on new rank function. Darehmiraki [15] discovered that it was developed based on the concept of $\alpha$ section, $\beta$ section, and left section of IFN . The proposed evaluation method is used to solve the problem of choosing a partner. Evaluation of partners by attributes is indicated using a triangular IFN. The proposed method can accurately evaluate the number of symmetric fuzzy sets that share the same core and different supports. Anushya et al. [16] transformed the fuzzy transmission problem into a definite problem by using the ranking method, used the VAM method to find the feasible solution, and used the MODI method to obtain the optimal solution for the initial solution. In this study, we introduce the concept of Value- and Ambiguity-based approach for solving the intuitionistic fuzzy transportation problem with total quantity discounts and with incremental quantity discounts. Pratihar et al. [20] discussed the interval type 2 fuzzy transmission problem. For this, the transportation costs, supply, and demand are represented by interval type 2 fuzzy numbers. Recently, Kumar et al. [21] discovered a simplified representation of a novel computational method for solving the Pythagorean purge transportation problem. Recently, Edalatpanah [23] developed a new model of data envelopment analysis based on new ranking functions of triangular neutrosophic numbers. Edalatpanah [24] introduced a new method called a neutrosophic structured element. Based on this approach, they proposed a multiattribute decision-making problem under NSE information. Pratihar et al. [20] solved a fuzzy transportation problem based on modified classical Vogel's approximation method, where the transportation cost, demand, and supply are represented by type 2 fuzzy sets.

Bagheri et al. [25] made the first attempt to solve the multiobjective fuzzy transportation problem using the fuzzy data analysis method. Ebrahimnejada et al. [26] discovered an effective solution to find the optimal weight of the fuzzy path by interval values. Ebrahimnejad [27] provided a new way to solve the fuzzy transfer problem (FTP). In this method, transportation cost, supply, and demand are represented by a nonnegative flat fuzzy number LR. Ebrahimnejad and Verdegay [28] have proposed an efficient computational solution approach for solving intuitionistic fuzzy transportation problems in which costs are triangular intuitionistic fuzzy numbers (TIFN) and availabilities and demands are taken as exact numerical values. To the best of our knowledge, there are no studies carried out on intuitionistic fuzzy transportation problem with total quantity discounts and incremental quantity discounts. The main objective of this study is to solve the new transportation problem with total quantity discounts and incremental quantity discounts in the intuitionistic fuzzy environment without using the ranking function.

The contributions of this paper are as follows:

(1) We introduce the intuitionistic fuzzy transportation problem with total quantity discounts and incremental quantity discounts.

(2) We developed a new approach called Value index and Ambiguity index to solve the above problem.

(3) Without using the ranking function, the intuitionistic fuzzy transportation problem is converted to two sub-problems.

(4) The proposed approach is illustrated with a numerical example.

The remaining paper is organized as follows: in Section 2, the basic preliminaries related to IFS and optimization are 
summarized briefly. In Section 3, the Value and Ambiguity of IFS and its properties are discussed. The mathematical formulation of the proposed model is presented in Section 4. Section 5 provides a numerical illustration of the proposed problem. The conclusion is drawn in the last section.

\section{Preliminaries}

This section describes some fundamental ideas relating to the intuitionistic fuzzy numbers and arithmetic operation of intuitionistic fuzzy numbers.

Definition 1 (see [1]). Let $x$ be the universe of discourse, then an intuitionistic fuzzy set $\widetilde{A}^{\mathrm{IFS}}$ in $X$ is given by the set of

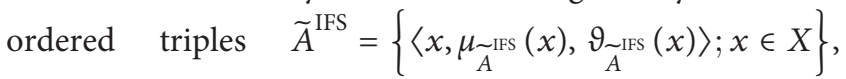

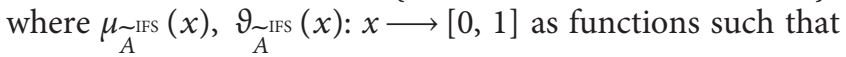
$0 \leq \mu_{A} \operatorname{IFS}(x)+\vartheta_{\mathcal{A}}^{\operatorname{IFS}}(x) \leq 1 \quad \forall x \in X$. For each $x$, the membership $\mu_{\mathcal{A}}$ IFS $(x)$ and the nonmembership $\vartheta_{\mathcal{A}}$ IFS $(x)$ represent the degree of membership and degree of nonmembership of the element $x \in X$ and $A \subset X$, respectively.

Definition 2 (intuitionistic fuzzy number (IFN) [1]). An IFN $\widetilde{A}^{\text {IFN }}$ is an intuitionistic fuzzy subset of the real line:

(i) Normal; i.e., there is any $x_{0} \in R$ such that $\mu_{A}^{\sim \text { IFN }}\left(x_{0}\right)=1, \vartheta_{A}^{\sim \text { IFN }}\left(x_{0}\right)=0$.

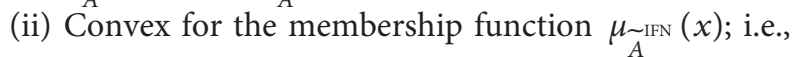

$$
\mu_{A}^{\sim \mathrm{IFN}}(x)\left(\lambda x_{1}+(1-\lambda) x_{2}\right) \geq \operatorname{Min}\left\{\mu_{A}^{\operatorname{IFN}}\left(x_{1}\right), \mu_{A}^{\sim \mathrm{IFN}}\left(x_{2}\right)\right\},
$$

for every $x_{1}, x_{2} \in R, \lambda \in[0,1]$.

(iii) Concave for the nonmembership function $\vartheta_{\widetilde{A}^{\text {IFN }}}(x)$; i.e.,

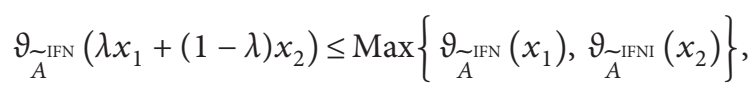

for every $x_{1}, x_{2} \in R, \lambda \in[0,1]$.

Definition 3 (trapezoidal intuitionistic fuzzy number (TrIFN) [1]). A trapezoidal intuitionistic fuzzy number $\tilde{A}^{\text {IFN }}=<\left(a_{2}, a_{3} \cdot a_{4} \cdot a_{5}\right) \omega_{\tilde{a}},\left(a_{1}, a_{3} \cdot a_{4} \cdot a_{6}\right)$, $\mathfrak{t}_{a}$, , where $a_{1} \leq a_{2} \leq a_{3} \leq a_{4} \leq a_{5} \leq a_{6}$ is a special intuitionistic fuzzy set on the real number set $R$, whose membership and nonmembership functions are defined as follows:

$$
\begin{aligned}
& \mu_{A}^{\sim \mathrm{IFN}}(x)= \begin{cases}0, & \text { if } x \leq a_{2}, \\
\frac{\left(x-a_{2}\right) \omega_{a}}{a_{3}-a_{2}}, & \text { if } a_{2} \leq x \leq a_{3}, \\
\frac{\omega_{a}^{\sim},}{\left(a_{5}-x\right) \omega_{a}} & \text { if } a_{3} \leq x \leq a_{4}, \\
a_{5}-a_{4} & \text { if } a_{4} \leq x \leq a_{5}, \\
0, & \text { if } x>a_{5},\end{cases} \\
& \vartheta_{\widetilde{A}} \operatorname{IFN}(x)= \begin{cases}1, & \text { if } x<a_{1}, \\
\frac{\left(x-a_{1}\right) \mathfrak{t}_{\tilde{a}}\left(a_{3}-x\right)}{a_{3}-a_{1}}, & \text { if } a_{1} \leq x \leq a_{3}, \\
\mathfrak{t}_{\tilde{a}}, & \text { if } a_{3} \leq x \leq a_{4}, \\
\frac{\left(1-\mathfrak{t}_{\tilde{a}}\right)\left(x-a_{4}\right)+\mathfrak{t}_{\tilde{a}}\left(a_{6}-a_{4}\right)}{a_{6}-a_{3}}, & \text { if } a_{1} \leq x \leq a_{3}, \\
1, & \text { if } x>a_{6} .\end{cases}
\end{aligned}
$$

The Values $\omega_{\mathfrak{a}}$ and $\mathfrak{t} \sim \widetilde{a}$ represent the maximum degree of membership and the minimum degree of nonmembership, respectively, so that $0 \leq \mu_{\mathcal{A}}^{\operatorname{IFN}}(x) \leq 1,0 \leq \vartheta_{\mathcal{A}} \sim_{\mathrm{IFN}}(x) \leq 1$; $0 \leq \mu_{A}^{-\mathrm{IFN}}(x)+\vartheta_{\sim_{A}^{\mathrm{IFN}}}(x) \leq 1 \quad \forall x \in X$; if the conditions are satisfied, Parameters $\omega_{a}$ and $\mathfrak{t}_{\tilde{a}}$ reflect TrIFN level of trust and level of uncertainty, respectively.

In addition, $\pi_{\tilde{a}}(x)=1-\mu_{A}^{\sim \mathrm{IFN}}(x)-\vartheta_{\widetilde{A}_{\mathrm{IFN}}}(x)$ is called the degree of indeterminacy of $x$ to $\widetilde{A}^{\mathrm{IFN}}$, or called the degree of hesitancy of $x$ to $\widetilde{A}^{\mathrm{IFN}}$.

If $a_{1} \geq 0$, then the $\operatorname{TrIFN} \widetilde{A}^{\text {IFN }}=<\left(a_{2}, a_{3}, a_{4}, a_{5}\right) \omega_{\tilde{a}},\left(a_{2}\right.$, $\left.a_{3}, a_{4}, a_{6}\right), \mathfrak{t} \sim \tilde{a}^{>}$is positive and is indicated by $\widetilde{A}^{\mathrm{IFN}}>0$.

Likewise, if $a_{1} \leq 0$, then the TrIFN $\widetilde{A}^{\mathrm{IFN}}=<\left(a_{2}, a_{3}, a_{4}, a_{5}\right)$ $\omega_{\widetilde{a}},\left(a_{1}, a_{3}, a_{4}, a_{6}\right), \mathfrak{t}_{\tilde{a}}$ negative and is indicated by $\widetilde{\widetilde{A}}_{\mathrm{IFN}}<0$.

If $\omega_{\tilde{a}}=1$ and $\mathfrak{t}_{\tilde{a}}=0$, then TrIFN is reduced to $\widetilde{A}^{\text {IFN }}=<$ $\left(a_{2}, a_{3}, a_{4}, a_{5}\right) 1,\left(a_{1}, a_{3}, a_{4}, a_{6}\right), 0>$, which is called the trapezoidal intuitionistic fuzzy number.

Since the TrIFN concept is generalization of trapezoidal fuzzy numbers, the arithmetic operation of TrIFNs can be defined as follows.

2.1. Arithmetic Operations of IFN. Let $\widetilde{A}^{\mathrm{IFN}}=<\left(a_{2}, a_{3}, a_{4}, a_{5}\right)$ $\omega \widetilde{a},\left(a_{1}, a_{3}, a_{4}, a_{6}\right), \mathfrak{t}_{\tilde{a}}>$ and $\widetilde{B}^{\mathrm{IFN}}=<\left(b_{2}, b_{3}, b_{4}, b_{5}\right) \omega_{-}^{-},\left(b_{1}\right.$, $\left.b_{3}, b_{4}, b_{6}\right), \mathfrak{t} \sim \vec{b}$ be two TrIFNs and $\lambda$ be a real number. The arithmetical operations are defined as follows[22]: 
(i) $\widetilde{A}^{\mathrm{IFN}}+\widetilde{B}^{\mathrm{IFN}}=<\left(a_{2}+b_{2}, a_{3}+b_{3}, a_{4}+b_{4}, a_{5}+b_{5}\right)$, $w,\left(a_{1}+b_{1}, a_{3}+b_{3}, a_{4}+b_{4}, a_{6}+b_{6}\right), u>$, where $w=\min \left\{\omega_{\tilde{a}}, \omega_{-}\right\}$and $u=\max \left\{\mathfrak{u}_{\tilde{a}}, \mathfrak{t}_{\tilde{b}}\right\}$

(ii) $\widetilde{A}^{\mathrm{IFN}}-\widetilde{B}^{\mathrm{IFN}}=$ by $<\left(a_{2}-b_{5}, a_{3}-b_{4}, a_{4}-b_{3}, a_{5}-b_{2}\right)$ $w,\left(a_{1}-b_{6}, a_{3}-b_{4}, a_{4}-b_{3}, a_{6}-b_{6}\right) u>$, where $w=\min \left\{\omega_{\tilde{a}}, \omega_{b}^{-}\right\}$and $u=\max \left\{\mathfrak{t}_{\tilde{a}}, \mathfrak{t}_{b}\right\}$

(iii) $\widetilde{A}^{\mathrm{IFN}} * \widetilde{B}^{\mathrm{IFN}}=<\left(a_{2} b_{2}, a_{3} b_{3}, a_{4} b_{4}, a_{5} b_{5}\right) w,\left(a_{1} b_{1}\right.$, $\left.a_{3} b_{3}, a_{4} b_{4}, a_{6} b_{6}\right) \mathcal{u}>$, where $w=\min \left\{\omega_{\tilde{a}}, \omega_{b}\right.$ and $u=\max \left\{\mathfrak{u}_{\tilde{a}}, \mathfrak{u t}_{\tilde{b}}\right\}$

(iv) $\left.\widetilde{A}^{\mathrm{IFN}} / \widetilde{B}^{\mathrm{IFN}}=<a_{1} / b_{6}, a_{3} / b_{4}, a_{4} / b_{3}, a_{5} / b_{2}\right), w\left(a_{1} / b_{5}\right.$, $\left.a_{3} / b_{4}, a_{4} / b_{2}, a_{5} / b_{1}\right) u>$, where $w=\min \left\{\omega_{-}, \omega_{b}\right\}$ and $u=\max \{\mathfrak{i} \tilde{a}, \mathfrak{t} \tilde{b}\}$

(v) $K \widetilde{A}^{\text {IFN }}=\left\{\begin{array}{l}\left\langle\left(K a_{2}, K a_{3}, K a_{4}, K a_{5}\right) \omega_{\widetilde{a}},\left(K a_{1}, K a_{3}, K a_{4}, K a_{6}\right), \mathfrak{t}_{\widetilde{a}}, \text { if } k>0\right. \\ \left\langle\left(K a_{5}, K a_{4}, K a_{3}, K a_{2}\right) \omega_{\widetilde{a}}^{-},\left(K a_{6}, K a_{3}, K a_{4}, K a_{1}\right), \mathfrak{t}_{\tilde{a}}, \text { if } k<0\right.\end{array}\right.$

Definition 4 (see [1]). The $\alpha$-cut of a membership function is a crisp set, which consists of elements of $\widetilde{A}^{\text {IFN }}$ having at least degree $\alpha$. It is denoted by $\widetilde{A}_{\alpha}^{\mathrm{IFN}}=\left[A_{1}^{\prime}(\alpha), A_{2}^{\prime}(\alpha)\right]$ and is defined mathematically as $\widetilde{A}_{\alpha}^{\mathrm{IFN}}=\left\{x: \mu_{\tilde{A}}(x) \geq \alpha, \mathbf{x} \in \mathbf{X}\right.$, $\alpha \in[0,1]$.

Let $\widetilde{A}^{\text {IFN }}=<\left(a_{2}, a_{3}, a_{4}, a_{5}\right) \omega_{\tilde{a}},\left(a_{1}, a_{3}, a_{4}, a_{6}\right), \mathfrak{t}_{\tilde{a}}>$ be a TrIFN, then

$$
\tilde{A}_{\alpha}^{\mathrm{IFN}}=\left[A_{1}^{\prime}(\alpha), A_{2}^{\prime}(\alpha)\right]=\left[a_{2}+\frac{\alpha}{\omega_{\tilde{a}}}\left(a_{3}-a_{1}\right), a_{5}+\frac{\alpha}{\omega_{\tilde{a}}}\left(a_{4}-a_{5}\right)\right.
$$

$\beta$-Cut is defined as follows:

$$
\begin{aligned}
A_{\beta}= & \left\{x: \vartheta_{\beta}(x) \leq \beta\right\}=\left[A_{1}^{\prime}(\beta), A_{2}^{\prime}(\beta)\right] \\
= & \left\{\frac { 1 } { ( \mathfrak { t } _ { \tilde { a } } - 1 ) } \left[\beta\left(a_{3}-a_{1}\right)+\mathfrak{t}_{\tilde{a}} a_{1}\right.\right. \\
& \left.-a_{3}\right], \frac{1}{\left(1-\mathfrak{t}_{\tilde{a}}\right)}\left[a_{6}\left(\boldsymbol{\beta}-\mathfrak{t}_{\tilde{a}}\right)+a_{4}(1-\boldsymbol{\beta})\right]
\end{aligned}
$$

$\alpha, \beta$-Cut is defined as follows:

$\widetilde{A}_{\boldsymbol{\alpha}, \beta}^{\mathrm{IFN}}=\left\{x: \underset{A}{\mu_{\text {IFN }}}(x) \geq \boldsymbol{\alpha}, \vartheta_{\mathcal{A}^{\mathrm{IFN}}}(x) \leq \beta, \boldsymbol{\alpha}+\boldsymbol{\beta} \leq 1 ; x \in X\right\}$.

\section{Value and Ambiguity of IFN}

This section describes the value and ambiguity of the intuitionistic fuzzy number.

Definition 5 (see [22]). Let $\widetilde{A}_{\alpha}^{\mathrm{IFN}}$ and $\widetilde{A}_{\beta}^{\mathrm{IFN}}$ be the $\alpha$-cut and, $\beta$-cut of an TrIFN $\widetilde{A}^{\text {IFN }}$, then the Value of the membership $\mu_{\tilde{A}}(x)$ and the nonmembership $\vartheta_{\widetilde{A}}(x)$ for $\widetilde{A}^{\text {IFN }}$ is defined as follows:

$$
\begin{aligned}
& V_{\mu}=\int_{0}^{1}\left[L_{\widetilde{A}}(\alpha)+R_{\widetilde{A}}(\alpha)\right] f(\alpha) \mathrm{d} \alpha, \\
& V_{\vartheta}=\int_{0}^{1}\left[L_{\widetilde{A}}(\beta)+R_{\widetilde{A}}(\beta)\right] g(\beta) \mathrm{d} \beta,
\end{aligned}
$$

respectively.
Definition 6 (see[22]). Let $\widetilde{A}_{\alpha}^{\mathrm{IFN}}$ and $\widetilde{A}_{\beta}^{\mathrm{IFN}}$ be the $\alpha-$ cut and $\beta$-cut of an TrIFN $\widetilde{A}^{\mathrm{IFN}}$, respectively, then the Ambiguity of the membership $\mu_{A}$ IFN $(x)$ and nonmembership $\vartheta_{\mathcal{A}}^{\text {IFN }}(x)$ for $\widetilde{A}^{\mathrm{IFN}}$ is defined as follows:

$$
\begin{aligned}
A_{\mu} & =\int_{0}^{1}\left[R_{\widetilde{A}}(\alpha)-L_{\widetilde{A}}(\alpha)\right] f(\alpha) \mathrm{d} \alpha, \\
A_{\vartheta} & =\int_{0}^{1}\left[R_{\tilde{A}}^{\prime}(\beta)-L_{\tilde{A}}^{\prime}(\beta)\right] g(\beta) \mathrm{d} \beta,
\end{aligned}
$$

respectively.

If $\tilde{A}^{\text {IFN }}=<\left(a_{2}, a_{3} \cdot a_{4} \cdot a_{5}\right) \omega_{\tilde{a}},\left(a_{1}, a_{3} \cdot a_{4} \cdot a_{6}\right), \mathfrak{t}_{\tilde{a}}>$ is a trapezoidal intuitionistic fuzzy number, then

(i) Value of the membership $\mu_{\tilde{A}}(x)$ and non-member$\operatorname{ship} \vartheta_{\tilde{A}}(x)$ for $\widetilde{A}^{\mathrm{IFN}}$ is

$$
\begin{aligned}
& V_{\mu}\left(\widetilde{A}^{\mathrm{IFN}}\right)=\frac{\omega^{2}}{6}\left[a_{2}+a_{5}+2 a_{3}+2 a_{4}\right], \\
& V_{9}\left(\widetilde{A}^{\mathrm{IFN}}\right)=\frac{\left(\begin{array}{c}
u \sim-1 \\
a
\end{array}\right)^{2}}{6}\left[a_{1}+a_{6}+2 a_{3}+2 a_{4}\right] .
\end{aligned}
$$

(ii) Ambiguity of the membership $\mu_{\tilde{A}}(x)$ and nonmembership $\vartheta_{\mathcal{A}}(x)$ for $\widetilde{A}^{\mathrm{IFN}}$ is

$$
\begin{aligned}
& A_{\mu}\left(\tilde{A}^{\mathrm{IFN}}\right)=\frac{\omega^{2}}{6}\left[a_{5}+2 a_{4}-a_{2}-2 a_{3}\right], \\
& A_{\vartheta}\left(\widetilde{A}^{\mathrm{IFN}}\right)=\frac{\left(\begin{array}{c}
1-u_{a} \\
a
\end{array}\right)^{2}}{6}\left[a_{6}-2 a_{3}-a_{1}+2 a_{4}\right] .
\end{aligned}
$$

Definition 7. Value index and Ambiguity index of IFN [22]). Let $\tilde{A}^{\text {IFN }}=<\left(a_{2}, a_{3}, a_{4}, a_{5}\right) \omega_{\tilde{a}},\left(a_{1}, a_{3}, a_{4}, a_{6}\right), \mathfrak{t}_{\tilde{a}}>$ be a TrIFN, then Value index and Ambiguity index of $\widetilde{A}^{\text {IFN }}$ are defined as follows:

$$
\begin{aligned}
& V_{\lambda}\left(\widetilde{A}^{\mathrm{IFN}}\right)=V_{\mu}\left(\widetilde{A}^{\mathrm{IFN}}\right)+(1-\lambda) V_{\vartheta}\left(\widetilde{A}^{\mathrm{IFN}}\right), \\
& A_{\lambda}\left(\tilde{A}^{\mathrm{IFN}}\right)=A_{\mu}\left(\widetilde{A}^{\mathrm{IFN}}\right)+(1-\lambda) A_{\vartheta}\left(\widetilde{A}^{\mathrm{IFN}}\right),
\end{aligned}
$$

respectively, where $\lambda \in[0,1]$ is a weight representing the decision-making preference information. Suppose

(i) $\lambda \in(0,0.5] \Rightarrow$ decision prefers uncertainty or negative feeling

(ii) $\lambda \in(0.5,1] \Rightarrow$ decision prefers certainty or positive feeling

(iii) $\lambda \in(0.5] \Rightarrow$ decision prefers between positive and negative feeling

Theorem 1 (see [22]). If $\omega_{\widetilde{a}}$ and $\mathfrak{t}_{\tilde{a}}$ represent the maximum degree of membership and minimum degree of membership 
function, respectively, then they satisfy the condition $0 \leq \omega_{\tilde{a}} \leq 1,0 \leq \mathfrak{t}_{\tilde{a}} \leq 1,0 \leq \omega_{\tilde{a}}+\mathfrak{t}_{\tilde{a}} \leq 1$.

Theorem 2 (see [22]). Let $\widetilde{A}^{I F N}=<\left(a_{2}, a_{3}, a_{4}, a_{5}\right) \omega_{\widetilde{a}},\left(a_{1}\right.$, $\left.a_{3}, a_{4}, a_{6}\right), \mathfrak{t} \tilde{a}_{a}>$ and $\widetilde{B}^{I F N}=<\left(b_{2}, b_{3}, b_{4}, b_{5}\right) \omega_{\tilde{b}},\left(b_{1}, b_{3}, b_{4}, b_{6}\right)$, $\mathfrak{u t}_{\vec{b}}>$ be two TrIFNs. If $a_{2}>b_{5}, \omega_{\tilde{a}}=\omega_{\tilde{b}}$, and $\mathfrak{t}_{\tilde{a}}=\mathfrak{t}_{\tilde{b}}$, then $\widetilde{A}^{I F N}>\widetilde{B}^{I F N}$.

Theorem 3 (see [22]). Let $\widetilde{A}^{I F N}=<\left(a_{2}, a_{3}, a_{4}, a_{5}\right) \omega_{\widetilde{a}},\left(a_{1}\right.$, $\left.a_{3}, a_{4}, a_{6}\right), \mathfrak{t}_{\widetilde{a}}>$ and $\widetilde{B}^{I F N}=<\left(b_{2}, b_{3}, b_{4}, b_{5}\right) \omega_{b^{-}},\left(b_{1}, b_{3}, b_{4}, b_{6}\right)$, $\mathfrak{u t}_{b}>$, and $\widetilde{C}^{I F N}=\left\langle\left(c_{2}, c_{3}, c_{4}, c_{5}\right) \omega_{c^{\prime}},\left(c_{1}, c_{3}, c_{4}, c_{6}\right), \mathfrak{t}_{c}>b e\right.$ three ITrFNs. If $\widetilde{A}^{I F N}>\widetilde{B}^{I F N}$, then $\widetilde{A}^{I F N}+\widetilde{C}^{I F N}>\widetilde{B}^{I F N}+\widetilde{C}^{I F N}$, where $\omega_{\tilde{a}}=\omega_{b}^{\sim}$ and $\mathfrak{t}_{\tilde{a}}=\mathfrak{t}_{\tilde{b}}$.

Theorem 4 (see [22]). Let $\tilde{A}^{I F N}=<\left(a_{2}, a_{3}, a_{4}, a_{5}\right) \omega_{\tilde{a}},\left(a_{1}\right.$, $\left.\left.a_{3}, a_{4}, a_{6}\right), \mathfrak{t}_{\widetilde{a}}\right\rangle, \widetilde{B}^{I F N}=\left\langle\left(b_{2}, b_{3}, b_{4}, b_{5}\right) \omega_{\tilde{b}},\left(b_{1}, b_{3}, b_{4}, b_{6}\right), \mathfrak{t}_{\tilde{b}}>\right.$ be two TrIFNs, then

(i) $V_{\mu}\left(\widetilde{A}^{I F N}+\widetilde{B}^{I F N}\right)=V_{\mu}\left(\widetilde{A}^{I F N}\right)+V_{\mu}\left(\widetilde{B}^{I F N}\right)$.

(ii) $V_{9}\left(\widetilde{A}^{I F N}+\widetilde{B}^{I F N}\right)=V_{9}\left(\widetilde{A}^{I F N}\right)+V_{\vartheta}\left(\widetilde{B}^{I F N}\right)$.

(iii) $V_{\mu}\left(\gamma \widetilde{A}^{I F N}\right)=\gamma V_{\mu}\left(\widetilde{A}^{I F N}\right)$.

(iv) $A_{\mu}\left(\widetilde{A}^{I F N}+\widetilde{B}^{I F N}\right)=A_{\mu}\left(\widetilde{A}^{I F N}\right)+A_{\mu}\left(\widetilde{B}^{I F N}\right)$.

(v) $A_{\vartheta}\left(\widetilde{A}^{I F N}+\widetilde{B}^{I F N}\right)=A_{\vartheta}\left(\widetilde{A}^{I F N}\right)+A_{\vartheta}\left(\widetilde{B}^{I F N}\right)$.

(vi) $A_{\mu}\left(\gamma \widetilde{A}^{I F N}\right)=\gamma A_{\mu}\left(\tilde{A}^{I F N}\right)$.

\section{Intuitionistic Fuzzy Transportation Problem with Quantity Discounts}

The intuitionistic fuzzy transportation problem (IFTP) with quantity discounts can be classified into two ways:

(i) Transportation problem with intuitionistic fuzzy quantity discounts (TPIFQD)

(ii) Transportation problem with incremental intuitionistic fuzzy quantity discounts (TPIIFQD)

Let $\widetilde{C}_{i j k}$ be the unit cost of shipment from the $i^{\text {th }}$ source to the $j^{\text {th }}$ destination with the $k^{\text {th }}$ price breakpoint and $X_{i j k}$ be the quantity shipped from the $i^{\text {th }}$ source to the $j^{\text {th }}$ destination with the $k^{\text {th }}$ price breakpoint. The cost structure (price breakpoints) under TPIFQD is as follows:

$$
\widetilde{C}_{i j k}= \begin{cases}\widetilde{C}_{i j 1}^{\mathrm{IFN}}, & \text { if } 0 \leq X_{i j k}<\widetilde{q}^{\mathrm{IFN}}, \\ \widetilde{C}_{i j 2}^{\mathrm{IFN}}, & \text { if } \widetilde{q}_{i j 1}^{\mathrm{IFN}} \leq X_{i j k}<\widetilde{q}^{\mathrm{IFN}}, \\ \widetilde{C}_{i j r}^{\mathrm{IFN}}, & \text { if } X_{i j k} \geq \widetilde{q}_{i j r-1}^{\mathrm{IFN}} .\end{cases}
$$

If the quantity is 0 to $\widetilde{q}_{i j 1}^{\mathrm{IFN}}$, then the cost $\widetilde{C}_{i j 1}$. If the quantity is $\widetilde{q}_{i j 1}^{\mathrm{IFN}}$ to $\widetilde{q}_{i j 2}^{\mathrm{IFN}}$, then the cost is $\widetilde{C}_{i j 2}$, and so on. This scheme is known as the TPIFQD scheme, the transportation cost of this model. Here, cost and quantity price breakpoints are represented by trapezoidal intuitionistic fuzzy numbers.

On the other hand, if the quantity is 0 to $\tilde{q}_{i j 1}^{\text {IFN }}$, then the cost is $\widetilde{C}_{i j 1}$. If the quantity is $\tilde{q}_{i j 1}^{\mathrm{IFN}}$ to $\tilde{q}_{i j 2}^{\mathrm{IFN}}$, then cost is \left.${\widetilde{\widetilde{q}_{i j 1}}}_{C_{i j 1}}^{\mathrm{IFN}} C_{i j k}-\widetilde{q}_{i j 2}^{\mathrm{IFN}}\right) \widetilde{C}_{i j 2}$, and so on. This scheme is called transportation problem with incremental intuitionistic fuzzy quantity discounts.

4.1. General Framework of Transportation Problem with Intuitionistic Fuzzy Quantity Discounts. Let $a_{i}$ be the capacity of source $i$, where $i=1,2,3, \ldots, m ; b_{j}$ is the demand of the destination $j=1,2, \ldots, n$. Let $r$ be the total number of price breakpoints in any given combination of source and destination; $\widetilde{C}_{i j k}$ be the cost per unit of shipping from the source $i$ to destination $j$ under the $k^{\text {th }}$ price break, $k=1,2, \ldots, r ; \tilde{q}_{i j k}^{\text {IFN }}$, the upper bound in the last price break, in any given cell can be either finite or infinite; $X_{i j k}$ be the number of units to be shipped from the source $i$ to the destination $j$ under the $k^{\text {th }}$ price breakpoint; and $\tilde{p}_{i j k}^{\mathrm{IFN}}$ is the price per unit of the $k^{\text {th }}$ price breakpoint from the source $i$ to the destination $j$. The tabular form of the proposed model is shown in Table 1.

4.2. Mathematical Formulation of TPIFQD (Model I). The total quantity discount is a unique discount for all units of the goods purchased. In order to minimize the total cost of shipment under intuitionistic fuzzy quantity discounts, let us define

$$
Y_{i j k}= \begin{cases}1, & \text { if } X_{i j k}>0, \\ 0, & \text { other wise. }\end{cases}
$$

Mathematical model of the transportation problem with intuitionistic fuzzy quantity discounts is

$$
\operatorname{minimize} \widetilde{Z}=\sum_{i=1}^{m} \sum_{j=1}^{n} \sum_{k=1}^{r} \widetilde{C}_{i j k} X_{i j k} \text {, }
$$

subject to

(a) $\sum_{j=1}^{n} \sum_{k=1}^{r} X_{i j k}=\tilde{a}_{i}, i=1, \ldots, m$ (row-wise and rowwise supply constraints)

(b) $\sum_{i=1}^{m} \sum_{k=1}^{r} X_{i j k}=\widetilde{b}_{j}, j=1, \ldots, n$ (column-wise and column-wise demand constraints)

(c) $\sum_{k=1}^{r} Y_{i j k} \leq 1, i=1, \ldots, m, j=1, \ldots, n$ (the constraints set assure that sharing is made below one and only Value break within any given arrangement of the starting point $i$ and end point $j$ )

(d) $X_{i j k} \leq\left(\tilde{q}_{i j k}^{\mathrm{IFN}}-1\right) Y_{i j k}, i=1, \ldots, m, j=1, \ldots, n, k=1$, $\ldots, r$ (the constrain set restricts the distribution of units below any Value break to the respective higher bound within the given arrangement of the source $i$ to endpoint $j$ )

(e) $X_{i j 1} \geq\left(\widetilde{q}_{i j 1}^{\mathrm{IFN}}-1\right) Y_{i j 2}, i=1, \ldots, m, j=1, \ldots, n$ (the constraints set assures that the units arrangement below any Value break is more than or equal to the respective lower bound within any given arrangement of the source $i$ and end point $j$ )

(f) $X_{i j k} \geq\left(\tilde{q}_{i j k}^{\mathrm{IFN}}-\tilde{q}_{i j(k-1)}^{\mathrm{IFN}}\right) Y_{i j(k+1)}, i=1, \ldots, m, j=1, \ldots$, $n, k=1, \ldots, r$

(g) $X_{i j k} \geq 0, Y_{i j k}=0$ or 1

4.3. Mathematical Formulation of TPIIFQD (Model II). Discount for certain interval is called incremental quantity discount. For example, suppose we purchase 100 units, if the 
TABLE 1: Tabular form of the proposed model.

\begin{tabular}{|c|c|c|c|c|c|c|}
\hline & & 1 & 2 & $j$ & $n$ & \\
\hline \multirow{9}{*}{ Source } & \multirow{4}{*}{1} & $0 \leq X_{111}<\tilde{q}_{111}^{\mathrm{IFN}}: \tilde{p}_{111}^{\mathrm{IFN}}$ & $0 \leq X_{121}<\widetilde{q}_{121}^{\mathrm{IFN}}: \widetilde{p}_{I F N}^{121}$ & & $0 \leq X_{1 n 1} \widetilde{q}_{1 n 1}^{\mathrm{IFN}}: \tilde{p}_{1 n 1}^{\mathrm{IFN}}$ & \\
\hline & & $\begin{array}{c}\tilde{q}_{111}^{\mathrm{IFN}} \leq X_{112}<\widetilde{q}_{112}^{\mathrm{IFN}}: \widetilde{p}_{111}^{\mathrm{IFN}} \\
\ldots\end{array}$ & $\tilde{q}_{121}^{\mathrm{IFN}} X_{122} \widetilde{q}_{122}^{\mathrm{IFN}}: \tilde{p}_{121}^{\mathrm{IFN}}$ & $\begin{array}{l}\cdots \\
\cdots\end{array}$ & $\tilde{q}_{1 n 1}^{\mathrm{IFN}} X_{1 n 2} \widetilde{q}_{1 n 2}^{\mathrm{IFN}}: \tilde{p}_{1 n 2}^{\mathrm{IFN}}$ & $a_{1}$ \\
\hline & & $\widetilde{q}_{11 r}^{\mathrm{IFN}} \leq X_{11 r}<\infty: \tilde{p}_{11 r}^{\mathrm{IFN}}$ & $\tilde{q}_{11(r-1)}^{\mathrm{IFN}} \leq \tilde{X}_{12 r}: \infty: \tilde{p}_{12 r}^{\mathrm{IFN}}$ & $\ldots$ & $\widetilde{q}_{1 m(r-1)} \leq X_{1 n r}<\infty: \widetilde{p}_{1 n r}^{\mathrm{IFN}}$ & \\
\hline & & $0 \leq X_{211}<\widetilde{q}_{211}^{\mathrm{IFN}}: \widetilde{p}_{211}^{\mathrm{IFN}}$ & $0 \leq X_{221}<\widetilde{q}_{221}^{\mathrm{IFN}}: \widetilde{p}_{221}^{\mathrm{IFN}}$ & & $0 \leq X_{2 n 1}<\widetilde{q}_{2 n 1}^{\mathrm{IFN}}: \widetilde{p}_{2 n 1}^{\mathrm{IFN}}$ & \\
\hline & \multirow[t]{2}{*}{2} & $\tilde{q}_{211}^{\mathrm{IFN}} \leq X_{212}<\tilde{q}_{212}^{\mathrm{IFN}}: \tilde{p}_{212}^{\mathrm{IFN}}$ & $\tilde{q}_{221}^{\mathrm{IFN}} X_{222} \tilde{q}_{222}^{\mathrm{IFN}}: \tilde{p}_{222}^{\mathrm{IFN}}$ & & $\tilde{q}_{2 n 1}^{\mathrm{IFN}} \leq X_{2 n 2} \widetilde{q}_{2 n 2}^{\mathrm{IFN}}: \tilde{p}_{2 n 2}^{\mathrm{IFN}}$ & $a_{2}$ \\
\hline & & $\tilde{q}_{21(r-1)}^{\mathrm{IFN}} \leq X_{21 r}<\infty: \widetilde{p}_{21 r}^{\mathrm{IFN}}$ & $\widetilde{q}_{22(r-1)}^{\mathrm{IFN}} \leq X_{22 r} \infty: \widetilde{p}_{22 r}^{\mathrm{IFN}}$ & & $\tilde{q}_{2 m(r-1)}^{\mathrm{IFN}} \leq X_{2 n r} \infty: \widetilde{p}_{2 n r}^{\mathrm{IFN}}$ & \\
\hline & $i$ & $0 \leq X_{m 11}<\widetilde{q}_{m 11}^{\mathrm{IFN}}: \widetilde{p}_{m 11}^{\mathrm{IFN}}$ & $0 \leq X_{m 21}<\widetilde{q}_{m 21}^{\mathrm{IFN}}: \tilde{p}_{m 21}^{\mathrm{IFN}}$ & & $0 \leq X_{m n 1}<\tilde{q}_{m n 1}^{\mathrm{IFN}}: \widetilde{p}_{m n 1}^{\mathrm{IFN}}$ & $a_{i}$ \\
\hline & \multirow[t]{2}{*}{$m$} & $\tilde{q}_{m 11}^{\mathrm{IFN}} \leq X_{m 12} \tilde{q}_{m 12}^{\mathrm{IFN}}: \tilde{p}_{m 12}^{\mathrm{IFN}}$ & $\tilde{q}_{m 21}^{\mathrm{IFN}} \leq X_{m 22} \widetilde{q}_{m 22}^{\mathrm{IFN}}: \widetilde{p}_{m 22}^{\mathrm{IFN}}$ & & $\tilde{q}_{m n 1}^{\mathrm{IFN}} X_{m n 2} \widetilde{q}_{m n 2}^{\mathrm{IFN}}: \tilde{p}_{m n 2}^{\mathrm{IFN}}$ & $a_{m}$ \\
\hline & & $\tilde{q}_{m 1(r-1)}^{\mathrm{IFN}} \leq X_{m 1 r}^{\cdots}<\infty: \tilde{p}_{m 1 r}^{\mathrm{IFN}}$ & $\tilde{q}_{m 2(r-1)}^{\mathrm{IFN}} \leq X_{m 2 r}^{\cdots}<\infty: \tilde{p}_{m 2 r}^{\mathrm{IFN}}$ & $b_{j}$ & $\tilde{q}_{m n(r-1)}^{\mathrm{IFN}} \leq X_{m n r} \infty: \widetilde{p}_{m n r}^{\mathrm{IFN}}$ & \\
\hline
\end{tabular}

unit cost is Rs. 15 for 90 units and reaming unit cost is Rs. 5, then, the total cost is Rs. 1400. In order to minimize the total shipping cost, the mathematical model of the transportation problem with incremental intuitionistic fuzzy quantity discounts is

$$
\operatorname{minimize} \quad \widetilde{Z}=\sum_{i=1}^{m} \sum_{j=1}^{n} \sum_{k=1}^{r} \widetilde{C}_{i j k} X_{i j k} \quad \text {, }
$$

subject to

(a) $\sum_{j=1}^{n} \sum_{k=1}^{r} X_{i j k}=\tilde{a}_{i}, i=1, \ldots, m$ (row-wise and rowwise supply constraints)

(b) $\sum_{i=1}^{n} \sum_{k=1}^{r} X_{i j k}=\widetilde{b}_{j}, j=1, \ldots, n$ (column-wise and column-wise demand constraints)

(c) $X_{i j 1} \leq\left(\widetilde{q}_{i j 1}^{\mathrm{IFN}}-1\right) Y_{i j 1}, i=1, \ldots, m, j=1, \ldots, n$

(d) $X_{i j k} \leq\left(\tilde{q}_{i j k}^{\mathrm{IFN}}-\widetilde{q}_{i j(k-1)}^{\mathrm{IFN}}\right) Y_{i j k} i=1, \ldots, m, j=1, \ldots, n$, $k=1, \ldots, r$ (the constraints limit the arrangement of units in any cell to their higher bound on the increment quantity within the given combination of the source $i$ to end point $j$ )

(e) $X_{i j 1} \geq\left(\widetilde{q}_{i j 1}^{\mathrm{IFN}}-1\right) Y_{i j 2}, i=1, \ldots, m, j=1, \ldots, n$

(f) $X_{i j k} \leq\left(\widetilde{q}_{i j k}^{\mathrm{IFN}}-\widetilde{q}_{i j(k-1)}^{\mathrm{IFN}}\right) Y_{i j(k+1)}, i=1, \ldots, m, j=1, \ldots$, $n, k=1, \ldots, r$ (for a given source $i$ to end point $j$, if the distribution is made with respect to the $(k+1)^{\text {th }}$ cost break $(k \geq 0)$, the distribution with respect to the $k^{\text {th }}$ cost break must be equal to the respective higher bound on the incremental quantity; i.e., $\left(\widetilde{q}_{i j k}-\widetilde{q}_{i j(k-1)}\right)$

(g) $X_{i j k} \geq 0, Y_{i j k}=0$ or 1

After modelling, the Value and Ambiguity of each IFN is computed and then the Value index and Ambiguity index are defined. Now, the IFTP model I is converted to two subproblems such as Value index problem and Ambiguity index problem, which are parametric linear programming problems with parameter $\lambda(0<\lambda<1)$. Similarly, model II is converted to Value index problem and Ambiguity index problem, which are parametric linear programming problems with parameter $\lambda$ $(0<\lambda<1)$. For different Values of $\lambda(0<\lambda<1)$, the parametric linear programming problems are converted to linear programming problems. LP problems have been solved in classical methods, which gives the Value and Ambiguity of the solution. The above method is explained using the following numerical example.

4.4. Numerical Example. A dairy firm has three plants located throughout a state. The supply, demand (in million of litres), and cost (in thousands of rupees) of shipping of milk from each plant to each distribution centre is given in $\mathrm{Ta}$ bles 2 and 3 .

The values in Table 3 are expressed as $\widetilde{q}_{i j k}: \widetilde{C}_{i j k}, i=1,2,3$, $j=1,2,3, k=1,2,3$. For example, in Table 3, the first row indicates the following: if the shipping quantity lies between $<(-0.5,0,1,2), 0.5,(-1,0,1,3) 0.3>$ and $<(16,18,20,22)$, $0.6(15,18,20,23), 0.3>$, then the shipping cost is $<(5,6,7$, 9), $0.6(4,6,7,10) 0.3>$; or, if the shipping quantity lies between $<(16,18,20,22), 0.6(15,18,20,23), 0.3>$ and $<(44$, $45,46,47), 0.6(43.45,46,48), 0.2>$, the cost is $<(4,5,6,7)$, $0.7(3,5,6,8,8) 0.3>$, and if the shipping quantity lies between < $(16,18,20,22), 0.8(15,18,22,23), 0.8>$ and < $(99.100,101,102), 0.6(98,100,101,103), 0.3>$, then the cost is $<(3,4,5,8), 0.8(2,4,5,10) 0.3>$. Similarly, the other price breakpoints and cost are given in Table 3.

The dairy firm wishes to determine how much should be the shipment from each milk plant to each distribution centre so that the total cost of the shipment is minimum.

4.4.1. Value of the Solution for Intuitionistic Fuzzy Quantity Discount and Incremental Intuitionistic Fuzzy Quantity Discount Transportation Problems. In order to solve Value of the solution for TPIFQD and TPIIFQD, first the Value of 
TABLE 2: Supply and demand.

\begin{tabular}{|c|c|c|c|c|}
\hline & Distribution centre 1 & Distribution centre 2 & Distribution centre 3 & Supply (in million litres ) \\
\hline Plant 1 & $\begin{aligned} \tilde{q}_{111}: & \widetilde{C}_{111} \\
\tilde{q}_{112}: & \widetilde{C}_{112} \\
\tilde{q}_{113}: & \widetilde{C}_{113}\end{aligned}$ & $\begin{aligned} \tilde{q}_{121} & : \widetilde{C}_{121} \\
\tilde{q}_{122}: & \widetilde{C}_{122} \\
\tilde{q}_{123}: & \widetilde{C}_{123}\end{aligned}$ & $\begin{array}{r}\tilde{q}_{131}: \widetilde{C}_{131} \\
\tilde{q}_{132}: \widetilde{C}_{132} \\
\tilde{q}_{133}: \widetilde{C}_{133}\end{array}$ & 45 \\
\hline Plant 2 & $\begin{array}{l}\tilde{q}_{211}: \widetilde{C}_{211} \\
\tilde{q}_{212}: \widetilde{C}_{212} \\
\tilde{q}_{213}: \widetilde{C}_{213}\end{array}$ & $\begin{array}{l}\tilde{q}_{221}: \widetilde{C}_{221} \\
\widetilde{q}_{222}: \widetilde{C}_{222} \\
\tilde{q}_{223}: \widetilde{C}_{223}\end{array}$ & $\begin{array}{l}\tilde{q}_{231}: \widetilde{C}_{231} \\
\widetilde{q}_{232}: \widetilde{C}_{232} \\
\tilde{q}_{233}: \widetilde{C}_{233}\end{array}$ & 45 \\
\hline Plant 3 & $\begin{array}{l}\widetilde{q}_{311}: \widetilde{C}_{311} \\
\widetilde{q}_{312}: \widetilde{C}_{312} \\
\widetilde{q}_{313}: \widetilde{C}_{313} \\
\end{array}$ & $\begin{array}{l}\widetilde{q}_{321}: \widetilde{C}_{321} \\
\widetilde{q}_{322}: \widetilde{C}_{322} \\
\widetilde{q}_{323}: \widetilde{C}_{323} \\
\end{array}$ & $\begin{array}{l}\widetilde{q}_{331}: \widetilde{C}_{331} \\
\widetilde{q}_{332}: \widetilde{C}_{332} \\
\widetilde{q}_{333}: \widetilde{C}_{333} \\
\end{array}$ & 30 \\
\hline Demand (in million litres) & 60 & 30 & 30 & 120 \\
\hline
\end{tabular}

TABLE 3: Cost and price break of the TP.

$\widetilde{q}_{11 k}: \widetilde{C}_{11 k}, k=1,2,3$

$<(-0.5,0,1,2), 0.5,(-1,0,1,3) 0.3>\leq X_{111}<(16.18,20,22), 0.6(15,18,20,23), 0.3>:<(5,6,7,9), 0.6(4,6,7,10) 0.3>$ $<(16,18,20,22), 0.6(15,18,20,23), 0.3 \leq X_{112}<(44,45,46,47), 0.6(43.45,46,48), 0.2>:<(4,5,6,7), 0.7(3,5,6,8,8) 0.3>$ $\left.<(16,18,20,22), 0.8(15,18,22,23), 0.8>\leq X_{113}<(99.100,101,102), 0.6(98,100,101,103), 0.3\right\rangle:<(3,4,5,8), 0.8(2,4,5,10) 0.3>$

$\widetilde{q}_{12 k}: \widetilde{C}_{12 k}, k=1,2,3$

$<(-0.5,0,1,2), 0.5,(-1,0,1,3) 0.3>\leq X_{121}<<(7,8,9,11), 0.6(6,8,9,12), 0.3>:<(6,7,8,10), 0.6(5,7,8,13) 0.3>$

$<(7,8,9,11), 0.6(6,8,9,12), 0.3>\leq X_{122}<(16,18,20,22), 0.6(15,18,20,23), 0.3>:<(5,6,7,9), 0.6(4,6,7,10) 0.3>$

$<(16,18,20,22), 0.6(15,18,20,23), 0.3>\leq X_{123}<<(99.100,101,102), 0.6(98,100,101,103), 0.3>:<(4,5,6,7), 0.7(3,5,6,8,8) 0.3>$

$\widetilde{q}_{13 k}: \widetilde{C}_{13 k}, k=1,2,3$

$<(-0.5,0,1,2), 0.5,(-1,0,1,3) 0.3>\leq X_{131}<<(4,5,6,7), 0.7(3,5,6,8,8) 0.3>:<(6,7,8,10), 0.6(5,7,8,13) 0.3>$

$<(4,5,6,7), 0.7(3,5,6,8,8) 0.3>\leq X_{132}<<(16.18,20,22), 0.6(15,18,20,23), 0.3>:<(6,7,8,10), 0.6(5,7,8,13) 0.3>$

$<(16.18,20,22), 0.6(15,18,20,23), 0.3>\leq X_{133}<<(99.100,101,102), 0.6(98,100,101,103), 0.3>:<(5,6,7,9), 0.6(4,6,7,10) 0.3>$

$\widetilde{q}_{21 k}: \widetilde{C}_{21 k}, k=1,2,3$

$<(-0.5,0,1,2), 0.5,(-1,0,1,3) 0.3>\leq X_{211}<<(44,45,46,47), 0.6(43.45,46,48), 0.2>:<(16.18,20,22), 0.6(15,18,20,23), 0.3>$

$<(44,45,46,47), 0.6(43.45,46,48), 0.2>\leq X_{212}<<(64,65,66,68), 0.6(63,65,66,69), 0.3>:<(6,7,8,10), 0.6(5,7,8,13) 0.3>$

$<(64,65,66,68), 0.6(63,65,66,69), 0.3>\leq X_{213}<<(99.100,101,102), 0.6(98,100,101,103), 0.3>:<(4,5,6,7), 0.7(3,5,6,8,8) 0.3>$

$\widetilde{q}_{22 k}: \widetilde{C}_{22 k}, k=1,2,3$

$<(-0.5,0,1,2), 0.5,(-1,0,1,3) 0.3>\leq X_{221}<<(16.18,20,22), 0.6(15,18,20,23), 0.3>:<(6,7,8,10), 0.6(5,7,8,13) 0.3>$

$<(16.18,20,22), 0.6(15,18,20,23), 0.3>\leq X_{222}<<(64,65,66,68), 0.6(63,65,66,69), 0.3>:<(5,6,7,9), 0.6(4,6,7,10) 0.3>$

$<(64,65,66,68), 0.6(63,65,66,69), 0.3>\leq X_{223}<<(99.100,101,102), 0.6(98,100,101,103), 0.3>:<(5,6,7,9), 0.6(4,6,7,10) 0.3>$

$\widetilde{q}_{23 k}: \widetilde{C}_{23 k}, k=1,2,3$

$<(-0.5,0,1,2), 0.5,(-1,0,1,3) 0.3>-\leq X_{231}-<<(6,7,8,10), 0.6(5,7,8,13) 0.3>:<(6,7,8,10), 0.6(5,7,8,13) 0.3>$

$<(6,7,8,10), 0.6(5,7,8,13) 0.3>\leq X_{232}<<(16.18,20,22), 0.6(15,18,20,23), 0.3>:<(4,5,6,7), 0.7(3,5,6,8,8) 0.3>$

$<(16.18,20,22), 0.6(15,18,20,23), 0.3>\leq X_{233}<<(99.100,101,102), 0.6(98,100,101,103), 0.3>:<(3,4,5,8), 0.8(2,4,5,10) 0.3>$

$\widetilde{q}_{31 k}: \widetilde{C}_{31 k}, k=1,2,3$

$<(-0.5,0,1,2), 0.5,(-1,0,1,3) 0.3>\leq X_{311}<<(6,7,8,10), 0.6(5,7,8,13) 0.3>:<(44,45,46,47), 0.6(43.45,46,48), 0.2>$

$<(6,7,8,10), 0.6(5,7,8,13) 0.3>\leq X_{312}<<(16.18,20,22), 0.6(15,18,20,23), 0.3>:<(16.18,20,22), 0.6(15,18,20,23), 0.3>$

$<(16.18,20,22), 0.6(15,18,20,23), 0.3>\leq X_{313}<<(99.100,101,102), 0.6(98,100,101,103), 0.3><(4,5,6,7), 0.7(3,5,6,8,8) 0.3>$

$\widetilde{q}_{32 k}: \widetilde{C}_{32 k}, k=1,2,3$

$<(-0.5,0,1,2), 0.5,(-1,0,1,3) 0.3>-\leq X_{321}-<<(3,4,5,8), 0.8(2,4,5,10) 0.3>:<(6,7,8,10), 0.6(5,7,8,13) 0.3>$

$<(3,4,5,8), 0.8(2,4,5,10) 0.3>\leq X_{322}<<(16.18,20,22), 0.6(15,18,20,23), 0.3>:<(5,6,7,9), 0.6(4,6,7,10) 0.3>$

$<(16.18,20,22), 0.6(15,18,20,23), 0.3>\leq X_{323}<(99.100,101,102), 0.6(98,100,101,103), 0.3>:<(3,4,5,8), 0.8(2,4,5,10) 0.3>$

$\widetilde{q}_{33 k}: \widetilde{C}_{33 k}, k=1,2,3$

$<(-0.5,0,1,2), 0.5,(-1,0,1,3) 0.3>\leq X_{331}<<(4,5,6,7), 0.7(3,5,6,8,8) 0.3>:<(6,7,8,10), 0.6(5,7,8,13) 0.3>$

$<(4,5,6,7), 0.7(3,5,6,8,8) 0.3>\leq X_{332}<<(44,45,46,47), 0.6(43.45,46,48), 0.2>:<(4,5,6,7), 0.7(3,5,6,8,8) 0.3>$

$<(44,45,46,47), 0.6(43.45,46,48), 0.2>\leq X_{333}<(99.100,101,102), 0.6(98,100,101,103), 0.3>:<(3,4,5,8), 0.8(2,4,5,10) 0.3>$

all IFNs is calculated. Then, the Value index is evaluated, which is shown in Table 4.

Case I. $(\lambda=0.25)$. Substituting $\lambda=0.25$ in the Value index in Table 4, we obtain the Value of the intuitionistic fuzzy cost and intuitionistic fuzzy price breakpoints, which is given in Table 5.

Model I: now, using the above Value of the intuitionistic fuzzy cost and intuitionistic fuzzy price breakpoints, the transportation problem with 
TABLE 4: TheValue index for TPIFQD and TPIIFQD.

$\widetilde{q}_{12 k}: \widetilde{C}_{12 k}, k=1,2,3$

$0.21 \lambda+(1-\lambda) 0.32 \leq X_{121}<3.12 \lambda+(1-\lambda) 4.24: 2.7 \lambda+(1-\lambda) 3.68$

$3.12 \lambda+(1-\lambda) 4.24 \leq X_{122}<6.84 \lambda+(1-\lambda) 9.31: 2.4 \lambda+(1-\lambda) 3.27$ $6.84 \lambda+(1-\lambda) 9.31 \leq X_{123}<36.18 \lambda+(1-\lambda) 64.32: 1.98 \lambda+(1-\lambda)$ 2.7

$\widetilde{q}_{13 k}: \widetilde{C}_{13 k}, k=1,2,3$

$0.21 \lambda+(1-\lambda) 0.32 \leq X_{121}<1.98 \lambda+(1-\lambda) 2.7: 3.12 \lambda+(1-\lambda) 4.24$ $1.98 \lambda+(1-\lambda) 2.7 \leq X_{122}<6.84 \lambda+(1-\lambda) 9.31: 2.7 \lambda+(1-\lambda) 3.68$ $6.84 \lambda+(1-\lambda) 9.31 \leq X_{123}<36.18 \lambda+(1-\lambda) 64.32: 2.4 \lambda+(1-\lambda)$ 3.27

$\widetilde{q}_{21 k}: \widetilde{C}_{21 k}, k=1,2,3$

$0.21 \lambda+(1-\lambda) 0.32 \leq X_{211}<0.3 \lambda+(1-\lambda) 0.75: 6.84 \lambda+(1-\lambda) 9.31$

$0.3 \lambda+(1-\lambda) 0.75 \leq X_{212}<23.64 \lambda+(1-\lambda) 32.18: 3.12 \lambda+(1-\lambda)$

4.24

$23.64 \lambda+(1-\lambda) 32.18 \leq X_{213}<36.18 \lambda+(1-\lambda) 64.32:$

$1.98 \lambda+(1-\lambda) 2.7$

$\widetilde{q}_{22 k}: \widetilde{C}_{22 k}, k=1,2,3$

$0.21 \lambda+(1-\lambda) 0.32 \leq X_{221}<6.84 \lambda+(1-\lambda) 9.31: 3.12 \lambda+(1-\lambda)$

4.24

$6.84 \lambda+(1-\lambda) 9.31 \leq X_{222}<23.64 \lambda+(1-\lambda) 32.18: 2.7 \lambda+(1-\lambda)$

3.68

$23.64 \lambda+(1-\lambda) 32.18 \leq X_{2123}<36.18 \lambda+(1-\lambda) 64.32: 2.4 \lambda+(1-$

ג) 3.27

$\tilde{q}_{23 k}: \widetilde{C}_{23 k}, k=1,2,3$

$0.21 \lambda+(1-\lambda) 0.32 \leq X_{231}<2.7 \lambda+(1-\lambda) 3.68: 3.12 \lambda+(1-\lambda) 4.24$

$2.7 \lambda+(1-\lambda) 3.68 \leq X_{232}<6.84 \lambda+(1-\lambda) 9.31: 1.98 \lambda+(1-\lambda) 2.7$

$6.84 \lambda+(1-\lambda) 9.31 \leq X_{233}<36.18 \lambda+(1-\lambda) 64.32: 1.74 \lambda+(1-\lambda)$ 2.45

$\widetilde{q}_{31 k}: \widetilde{C}_{31 k}, k=1,2,3$

$0.21 \lambda+(1-\lambda) 0.32 \leq X_{311}<3.12 \lambda+(1-\lambda) 4.24: 0.3 \lambda+(1-\lambda) 0.75$

$3.12 \lambda+(1-\lambda) 4.24 \leq X_{312}<6.84 \lambda+(1-\lambda) 9.31: 6.84 \lambda+(1-\lambda)$

9.31

$6.84 \lambda+(1-\lambda) 9.31 \leq X_{312}<36.18 \lambda+(1-\lambda) 64.32: 3.12 \lambda+(1-\lambda)$

4.24

$\widetilde{q}_{32 k}: \widetilde{C}_{32 k}, k=1,2,3$

$0.21 \lambda+(1-\lambda) 0.32 \leq X_{321}<1.98 \lambda+(1-\lambda) 2.7: 3.12 \lambda+(1-\lambda) 4.24$

$1.98 \lambda+(1-\lambda) 2.7 \leq X_{322}<6.84 \lambda+(1-\lambda) 9.31: 1.98 \lambda+(1-\lambda) 2.7$

$6.84 \lambda+(1-\lambda) 9.31 \leq X_{323}<36.18 \lambda+(1-\lambda) 64.32: 1.74 \lambda+(1-\lambda)$ 2.45

$\widetilde{q}_{33 k}: \widetilde{C}_{33 k}, k=1,2,3 \widetilde{C}_{33}$

$0.21 \lambda+(1-\lambda) 0.32 \leq X_{331}<1.98 \lambda+(1-\lambda) 2.7: 3.12 \lambda+(1-\lambda) 4.24$

$1.98 \lambda+(1-\lambda) 2.7 \leq X_{332}<0.3 \lambda+(1-\lambda) 0.75: 1.98 \lambda+(1-\lambda) 2.7$

$0.3 \lambda+(1-\lambda) 0.75 \leq X_{333}<36.18 \lambda+(1-\lambda) 64.32: 1.74 \lambda+(1-\lambda)$

2.45

intuitionistic fuzzy quantity discounts is converted to the following linear programming problem:

$$
\begin{aligned}
\text { Minimize } & 3.4 x_{111}+2.7 x_{112}+2.1 x_{113}+3.8 x_{121}+3.4 x_{122} \\
& +2.7 x_{123}+4.42 x_{131}+3.8 x_{132}+3.4 x_{133} \\
& +14.58 x_{211}+4.42 x_{212}+2.7 x_{213}+4.42 x_{221} \\
& +3.8 x_{222}+3.4 x_{223}+4.42 x_{231}+2.7 x_{232} \\
& +2.1 x_{233}+25.94 x_{311}+14.58 x_{312}+4.42 x_{313} \\
& +4.42 x_{321}+3.4 x_{322}+2.1 x_{323}+4.42 x_{331} \\
& +2.7 x_{332}+2.1 x_{333}
\end{aligned}
$$

Subject to $X_{111}+X_{112}+X_{113}+X_{121}+X_{122}+X_{123}$

$+X_{131}+X_{132}+X_{133}=45$

$X_{211}+X_{212}+X_{213}+X_{221}+X_{222}+X_{223}$

$+X_{231}+X_{232}+X_{233}=45$;

$X_{311}+X_{312}+X_{313}+X_{321}+X_{322}+X_{323}$

$+X_{331}+X_{332}+X_{333}=30$

$X_{111}+X_{112}+X_{113}+X_{211}+X_{212}+X_{213}$

$+X_{311}+X_{312}+X_{313}=60$;

$X_{121}+X_{122}+X_{123}+X_{221}+X_{222}+X_{223}$

$+X_{321}+X_{322}+X_{323}=30$;

$X_{131}+X_{132}+X_{133}+X_{231}+X_{232}+X_{233}$

$+X_{331}+X_{332}+X_{333}=30$;

$Y_{111}+Y_{112}+Y_{113} \leq 1 ; Y_{121}+Y_{122}+Y_{123} \leq 1 ;$

$Y_{131}+Y_{132}+Y_{133} \leq 1 ; Y_{211}+Y_{212}+Y_{213} \leq 1$;

$Y_{221}+Y_{222}+Y_{223} \leq 1 ; Y_{231}+Y_{232}+Y_{233} \leq 1$;

$Y_{311}+Y_{312}+Y_{313} \leq 1 ; Y_{321}+Y_{322}+Y_{323} \leq 1$;

$Y_{331}+Y_{332}+Y_{333} \leq 1 ;-13.58 Y_{111}+X_{111} \leq 0$;

$-24.94 Y_{112}+X_{112} \leq 0 ;-77.74 Y_{113}+X_{113} \leq 0$;

$-3.42 Y_{121}+X_{121} \leq 0 ;-13.58 Y_{122}+X_{122} \leq 0$;

$-77.74 Y_{123}+X_{123} \leq 0 ;-1.7 Y_{131}+X_{131} \leq 0$;

$-13.58 Y_{132}+X_{132} \leq 0 ;-77.74 Y_{133}+X_{133} \leq 0$;

$-24.94 Y_{211}+X_{211} \leq 0 ;-74.8 Y_{212}+X_{212} \leq 0$;

$-77.74 Y_{213}+X_{213} \leq 0 ;-13.58 Y_{221}+X_{221} \leq 0$;

$-74.8 Y_{222}+X_{222} \leq 0 ;-77.74 Y_{223}+X_{223} \leq 0$;

$-2.8 Y_{231}+X_{231} \leq 0 ;-13.58 Y_{232}+X_{232} \leq 0$;

$-77.74 Y_{233}+X_{233} \leq 0 ;-3.42 Y_{311}+X_{311} \leq 0$;

$-13.58 Y_{312}+X_{312} \leq 0 ;-77.74 Y_{313}+X_{313} \leq 0$;

$-1.1 Y_{321}+X_{321} \leq 0 ;-13.58 Y_{322}+X_{322} \leq 0$;

$-77.74 Y_{323}+X_{323} \leq 0 ;-2.8 Y_{331}+X_{311} \leq 0$;

$-13.58 Y_{332}+X_{332} \leq 0 ;-77.74 Y_{333}+X_{333} \leq 0$;

$-14.58 Y_{112}+X_{112} \geq 0 ;-25.94 Y_{113}+X_{113} \geq 0$;

$-4.42 Y_{122}+X_{122} \geq 0 ;-14.58 Y_{123}+X_{123} \geq 0$;

$-2.7 Y_{132}+X_{132} \geq 0 ;-14.58 Y_{133}+X_{133} \geq 0$;

$-25.942 Y_{212}+X_{212} \geq 0 ;-75.8 Y_{213}+X_{213} \geq 0$;

$-14.58 Y_{222}+X_{222} \geq 0 ;-75.8 Y_{223}+X_{223} \geq 0$;

$-3.8 Y_{232}+X_{232} \geq 0 ;-14.58 Y_{233}+X_{233} \geq 0$;

$-4.42 Y_{312}+X_{312} \geq 0 ;-14.58 Y_{313}+X_{313} \geq 0$;

$-2.1 Y_{322}+X_{322} \geq 0 ;-14.58 Y_{323}+X_{323} \geq 0$;

$-3.8 Y_{332}+X_{332} \geq 0$;

$-14.58 Y_{333}+X_{333} \geq 0$;

where $X_{i j k} \geq 0, Y_{i j k} \geq 0$ or $1 ; i=1,2,3 ; j=1,2,3 ; k=1,2,3$. Model II: similarly, using the Value of the intuitionistic fuzzy cost and intuitionistic fuzzy price breakpoints in Table 5, the transportation problem with incremental intuitionistic fuzzy quantity discounts is converted to the following linear programming problem: 
TABLE 5: Value of the IF cost and IF price breakpoints when $\lambda=0.25$.

\begin{tabular}{|c|c|c|c|c|}
\hline \multicolumn{4}{|c|}{$\lambda=0.25$} & Supply \\
\hline \multirow{10}{*}{ Demand } & $0.28 \leq X_{111}<14.58: 3.4$ & $0.28 \leq X_{121}<4.42: 3.8$ & $0.28 \leq X_{131}<2.7: 4.4$ & \\
\hline & $14.58 \leq X_{112}<25.94: 2.7$ & $4.42=X_{122}<14.38: 3.4$ & $2.7=X_{132}<14.58: 3.8$ & 45 \\
\hline & $25.94 \leq X_{113}<77.74: 2.1$ & $14.38=X_{123}<77.74: 2.7$ & $14.58 \leq X_{133}<77.74: 3.4$ & \\
\hline & $0.28=X_{211}<25.94: 14.58$ & $0.28=X_{221}<14.58: 4.42$ & $0.28=X_{231}<3.8: 4.42$ & \\
\hline & $25.98=X_{212}<75.8: 4.42$ & $14.58=X_{222}<75.8: 3.8$ & $3.8=X_{232}<14.58: 2.7$ & 45 \\
\hline & $75.8=X_{213}<77.74: 2.7$ & $75.8=X_{223}<77.74: 3.4$ & $4.58=X_{233}<77.74: 2.1$ & \\
\hline & $0.28=X_{311}<4.42: 25.94$ & $0.28=X_{321}<2.1: 4.42$ & $0.28=X_{331}<3.8: 4.42$ & \\
\hline & $4.42=X_{312}<14.58: 14.58$ & $2.1=X_{322}<14.58: 3.4$ & $3.8=X_{332}<14.58: 2.7$ & 30 \\
\hline & $14.58=X_{313}<77.74: 4.42$ & $14.58=X_{323}<77.74: 2.1$ & $14.58=X_{333}<77.74: 2.1$ & \\
\hline & 60 & 30 & 30 & 120 \\
\hline
\end{tabular}

$$
\begin{aligned}
& \text { Minimize } \quad 3.4 x_{111}+2.7 x_{112}+2.1 x_{113}+3.8 x_{121}+3.4 x_{122}+2.7 x_{123}+4.42 x_{131} \\
& +3.8 x_{132}+3.4 x_{133}+14.58 x_{211}+4.42 x_{212}+2.7 x_{213}+4.42 x_{221}+3.8 x_{222} \\
& +3.4 x_{223}+4.42 x_{231}+2.7 x_{232}+2.1 x_{233}+25.94 x_{311}+14.58 x_{312}+4.42 x_{313} \\
& +4.42 x_{321}+3.4 x_{322}+2.1 x_{323}+4.42 x_{331}+2.7 x_{332}+2.1 x_{333} \\
& \text { Subject to } X_{111}+X_{112}+X_{113}+X_{121}+X_{122}+X_{123}+X_{131}+X_{132}+X_{133}=45 \text {; } \\
& X_{211}+X_{212}+X_{213}+X_{221}+X_{222}+X_{223}+X_{231}+X_{232}+X_{233}=45 \\
& X_{311}+X_{312}+X_{313}+X_{321}+X_{322}+X_{323}+X_{331}+X_{332}+X_{333}=30 \\
& X_{111}+X_{112}+X_{113}+X_{211}+X_{212}+X_{213}+X_{311}+X_{312}+X_{313}=60 \\
& X_{121}+X_{122}+X_{123}+X_{221}+X_{222}+X_{223}+X_{321}+X_{322}+X_{323}=30 \\
& X_{131}+X_{132}+X_{133}+X_{231}+X_{232}+X_{233}+X_{331}+X_{332}+X_{333}=30 \text {; } \\
& -13.58 Y_{111}+X_{111} \leq 0 ;-11.36 Y_{112}+X_{112} \leq 0 \text {; } \\
& -77.74 Y_{113}+X_{113} \leq 0 ;-3.42 Y_{121}+X_{121} \leq 0 \text {; } \\
& -10.16 Y_{122}+X_{122} \leq 0 ;-77.74 Y_{123}+X_{123} \leq 0 \text {; } \\
& -1.7 Y_{131}+X_{131} \leq 0 ;-11.88 Y_{132}+X_{132} \leq 0 \text {; } \\
& -77.74 Y_{133}+X_{133} \leq 0 ;-24.94 Y_{211}+X_{211} \leq 0 \text {; } \\
& -49.86 Y_{212}+X_{212} \leq 0 ;-77.74 Y_{213}+X_{213} \leq 0 \text {; } \\
& -13.58 Y_{221}+X_{221} \leq 0 ;-61.22 Y_{222}+X_{222} \leq 0 \text {; } \\
& -77.74 Y_{223}+X_{223} \leq 0 ;-2.8 Y_{231}+X_{231} \leq 0 \text {; } \\
& -10.78 Y_{232}+X_{232} \leq 0 ;-77.74 Y_{233}+X_{233} \leq 0 \text {; } \\
& -43.42 Y_{311}+X_{311} \leq 0 ;-10.16 Y_{312}+X_{312} \leq 0 \text {; } \\
& -77.74 Y_{313}+X_{313} \leq 0 ;-1.1 Y_{321}+X_{321} \leq 0 \text {; } \\
& -12.48 Y_{322}+X_{322} \leq 0 ;-77.74 Y_{323}+X_{323} \leq 0 \text {; } \\
& -2.8 Y_{331}+X_{311} \leq 0 ;-10.78 Y_{332}+X_{332} \leq 0 \text {; } \\
& -77.74 Y_{333}+X_{333} \leq 0 ;-13.58 Y_{112}+X_{111} \geq 0 \text {; } \\
& -11.36 Y_{113}+X_{112} \geq 0 ;-3.42 Y_{122}+X_{121} \geq 0 \text {; } \\
& -10.16 Y_{123}+X_{122} \geq 0 ;-1.7 Y_{132}+X_{131} \geq 0 \text {; } \\
& -11.88 Y_{133}+X_{132} \geq 0 ;-24.94 Y_{212}+X_{211} \geq 0 \text {; } \\
& -49.86 Y_{213}+X_{212} \geq 0 ;-13.58 Y_{222}+X_{221} \geq 0 \text {; } \\
& -61.22 Y_{223}+X_{222} \geq 0 ;-2.8 Y_{232}+X_{231} \geq 0 \text {; } \\
& -10.78 Y_{233}+X_{232} \geq 0 ;-3.42 Y_{312}+X_{311} \geq 0 \text {; } \\
& -10.16 Y_{313}+X_{312} \geq 0 ;-1.1 Y_{322}+X_{321} \geq 0 \text {; } \\
& -12.48 Y_{323}+X_{322} \geq 0 ;-2.8 Y_{332}+X_{331} \geq 0 \text {; } \\
& -10.78 Y_{333}+X_{332} \geq 0 \text {; }
\end{aligned}
$$

where $X_{i j k} \geq 0, Y_{i j k} \geq 0$ or $1 ; i=1,2,3 ; j=1,2,3 ; k=1,2,3$.

Case II. $(\lambda=0.50)$. Substituting $\lambda=0.50$ in the Value index in Table 4, we obtain the Value of the intuitionistic fuzzy cost and intuitionistic fuzzy price breakpoints, which is given in Table 6 .
Model I: now, using the above Value of the intuitionistic fuzzy cost and intuitionistic fuzzy price breakpoints, the transportation problem with intuitionistic fuzzy quantity discounts is converted to the following linear programming problem: 
TABLE 6: Value of the IF cost and IF price breakpoints when $\lambda=0.50$.

\begin{tabular}{cccc}
\hline & $\lambda=0.50$ & & \\
\hline & & & \\
& $0.24 \leq X_{111}<9.18: 2.8$ & $0.24 \leq X_{121}<3.4: 3.18$ & $0.24 \leq X_{131}<2.7: 3.4$ \\
& $9.18 \leq X_{112}<22.75: 2.7$ & $3.4=X_{122}<9.18: 2.8$ & $2.7 \leq X_{132}<9.18: 3.18$ \\
& $22.75=X_{113}<73.16: 1.7$ & $9.18=X_{123}<73.16: 2.7$ & $9.18=X_{133}<73.16: 2.8$ \\
Demand & $0.24=X_{211}<22.75: 9.18$ & $0.24=X_{221}<9.18: 3.4$ & $0.24=X_{231}<3.18: 3.4$ \\
& $22.75=X_{212}<43.91: 3.4$ & $9.18=X_{222}<43.91: 3.18$ & $3.18=X_{232}<9.18: 2.7$ \\
& $43.91=X_{213}<73.16: 2.7$ & $43.91=X_{223}<73.16: 2.8$ & $9.18=X_{233}<73.16: 1.7$ \\
& $0.24=X_{311}<3.4: 22.75$ & $0.24=X_{321}<1.7: 3.4$ & $0.24=X_{331}<3.18: 3.4$ \\
$3.4=X_{312}<9.18: 9.18$ & $1.7=X_{322}<9.18: 2.8$ & $3.18=X_{332}<9.18: 2.7$ & 30 \\
$9.18=X_{313}<73.16: 3.4$ & $9.18=X_{323}<73.16: 1.7$ & $9.18=X_{333}<73.16: 1.7$ & 30 \\
60 & 30 & 120 \\
\hline
\end{tabular}

Minimize $2.8 x_{111}+2.7 x_{112}+1.7 x_{113}+3.18 x_{121}+2.8 x_{122}+2.7 x_{123}+3.4 x_{131}+3.18 x_{132}+2.8 x_{133}+9.18 x_{211}$ $+3.4 x_{212}+2.7 x_{213}+3.4 x_{221}+3.18 x_{222}+2.8 x_{223}+3.4 x_{231}+2.7 x_{232}+1.7 x_{233}+22.75 x_{311}$ $+9.18 x_{312}+3.4 x_{313}+3.4 x_{321}+2.8 x_{322}+1.7 x_{323}+3.4 x_{331}+2.7 x_{332}+1.7 x_{333}$

Subject to $X_{111}+X_{112}+X_{113}+X_{121}+X_{122}+X_{123}+X_{131}+X_{132}+X_{133}=45$

$X_{211}+X_{212}+X_{213}+X_{221}+X_{222}+X_{223}+X_{231}+X_{232}+X_{233}=45 ;$

$X_{311}+X_{312}+X_{313}+X_{321}+X_{322}+X_{323}+X_{331}+X_{332}+X_{333}=30$

$X_{111}+X_{112}+X_{113}+X_{211}+X_{212}+X_{213}+X_{311}+X_{312}+X_{313}=60$

$X_{121}+X_{122}+X_{123}+X_{221}+X_{222}+X_{223}+X_{321}+X_{322}+X_{323}=30 ;$

$Y_{111}+Y_{112}+Y_{113} \leq 1 ; Y_{121}+Y_{122}+Y_{123} \leq 1 ;$

$Y_{131}+Y_{132}+Y_{133} \leq 1 ; Y_{211}+Y_{212}+Y_{213} \leq 1 ;$

$Y_{221}+Y_{222}+Y_{223} \leq 1 ; Y_{231}+Y_{232}+Y_{233} \leq 1 ;$

$Y_{311}+Y_{312}+Y_{313} \leq 1 ; Y_{321}+Y_{322}+Y_{323} \leq 1 ;$

$Y_{331}+Y_{332}+Y_{333} \leq 1 ;-8.18 Y_{111}+X_{111} \leq 0$;

$-21.75 Y_{112}+X_{112} \leq 0 ;-73.16 Y_{113}+X_{113} \leq 0$;

$-2.4 Y_{121}+X_{121} \leq 0 ;-8.18 Y_{122}+X_{122} \leq 0$;

$-73.16 Y_{123}+X_{123} \leq 0 ;-1.7 Y_{131}+X_{131} \leq 0$;

$-8.18 Y_{132}+X_{132} \leq 0 ;-73.16 Y_{133}+X_{133} \leq 0$;

$-21.75 Y_{211}+X_{211} \leq 0 ;-42.91 Y_{212}+X_{212} \leq 0$;

$-73.16 Y_{213}+X_{213} \leq 0 ;-8.18 Y_{221}+X_{221} \leq 0$;

$-42.91 Y_{222}+X_{222} \leq 0 ;-73.16 Y_{223}+X_{223} \leq 0$;

$-2.18 Y_{231}+X_{231} \leq 0 ;-8.18 Y_{232}+X_{232} \leq 0$;

$-73.16 Y_{233}+X_{233} \leq 0 ;-2.4 Y_{311}+X_{311} \leq 0$;

$-8.18 Y_{312}+X_{312} \leq 0 ;-73.16 Y_{313}+X_{313} \leq 0$;

$-0.7 Y_{321}+X_{321} \leq 0 ;-8.18 Y_{322}+X_{322} \leq 0$;

$-73.16 Y_{323}+X_{323} \leq 0 ;-2.18 Y_{331}+X_{311} \leq 0$;

$-8.18 Y_{332}+X_{332} \leq 0 ;-73.16 Y_{333}+X_{333} \leq 0$;

$-9.18 Y_{112}+X_{112} \geq 0 ;-22.75 Y_{113}+X_{113} \geq 0$;

$-3.4 Y_{122}+X_{122} \geq 0 ;-9.18 Y_{123}+X_{123} \geq 0$;

$-2.7 Y_{132}+X_{132} \geq 0 ;-9.18 Y_{133}+X_{133} \geq 0$;

$-22.75 Y_{212}+X_{212} \geq 0 ;-43.91 Y_{213}+X_{213} \geq 0$;

$-9.18 Y_{222}+X_{222} \geq 0 ;-43.91 Y_{223}+X_{223} \geq 0$;

$-3.18 Y_{232}+X_{232} \geq 0 ;-9.18 Y_{233}+X_{233} \geq 0$;

$-3.4 Y_{312}+X_{312} \geq 0 ;-9.18 Y_{313}+X_{313} \geq 0$;

$-1.7 Y_{322}+X_{322} \geq 0 ;-9.18 Y_{323}+X_{323} \geq 0$;

$-3.18 Y_{332}+X_{332} \geq 0 ;-9.18 Y_{333}+X_{333} \geq 0$;

where $X_{i j k} \geq 0, Y_{i j k} \geq 0$ or $1 ; i=1,2,3 ; j=1,2,3 ; k=1,2,3$. Model II: similarly, using the Value of the intuitionistic fuzzy cost and intuitionistic fuzzy price breakpoints in
Table 7, the transportation problem with intuitionistic fuzzy incremental quantity discounts is converted to the following linear programming problem: 
TABLe 7: Value of the IF cost and IF price breakpoints when $\lambda=0.75$.

\begin{tabular}{cccc}
\hline & $\lambda=0.75$ & \\
\hline $0.19 \leq X_{111}<12.97: 2.7$ & $0.19 \leq X_{121}<2.8: 2.94$ & $0.19 \leq X_{131}<2.7: 2.94$ \\
& $12.97 \leq X_{112}<19.57: 2.1$ & $2.8 \leq X_{122}<12.97: 2.7$ & $2.7 \leq X_{132}<12.97: 2.8$ \\
$19.57=X_{113}<68.3: 1.44$ & $12.97 \leq X_{123}<68.83: 2.1$ & $12.97=X_{133}<68.83: 2.1$ \\
& $0.19=X_{211}<19.57: 12.97$ & $0.19=X_{221}<12.97: 2.94$ & $0.19=X_{231}<2.94: 2.8$ \\
Demand & $19.57=X_{212}<41.3: 2.8$ & $12.97=X_{222}<41.3: 2.8$ & $2.94 \leq X_{232}<12.97: 2.7$ \\
& $41.3=X_{213}<68.83: 2.7$ & $41.3=X_{223}<68.83: 2.1$ & $12.97=X_{233}<68.83: 1.44$ \\
& $0.19=X_{311}<2.8: 19.57$ & $0.19=X_{321}<1.44: 2.8$ & $0.19=X_{331}<2.94: 2.8$ \\
& $2.8=X_{312}<12.97: 12.97$ & $1.44=X_{322}<12.97: 2.1$ & $2.94=X_{332}<12.97: 2.7$ \\
& $12.97=X_{313}<68.83: 2.8$ & $12.97=X_{323}<68.83: 1.44$ & $12.97=X_{333}<68.83: 1.44$ \\
60 & 3330 & 30
\end{tabular}

Minimize $2.8 x_{111}+2.7 x_{112}+1.7 x_{113}+3.18 x_{121}+2.8 x_{122}+2.7 x_{123}+3.4 x_{131}+3.18 x_{132}+2.8 x_{133}+9.18 x_{211}+3.4 x_{212}$ $+2.7 x_{213}+3.4 x_{221}+3.18 x_{222}+2.8 x_{223}+3.4 x_{231}+2.7 x_{232}+1.7 x_{233}+22.75 x_{311}+9.18 x_{312}+3.4 x_{313}$ $+3.4 x_{321}+2.8 x_{322}+1.7 x_{323}+3.4 x_{331}+2.7 x_{332}+1.7 x_{333}$

Subject to $X_{111}+X_{112} X_{113}+X_{121}+X_{122}+X_{123}+X_{131}+X_{132}+X_{133}=45$

$X_{211}+X_{212}+X_{213}+X_{221}+X_{222}+X_{223}+X_{231}+X_{232}+X_{233}=45 ;$

$X_{311}+X_{312}+X_{313}+X_{321}+X_{322}+X_{323}+X_{331}+X_{332}+X_{333}=30 ;$

$X_{111}+X_{112}+X_{113}+X_{211}+X_{212}+X_{213}+X_{311}+X_{312}+X_{313}=60 ;$

$X_{121}+X_{122}+X_{123}+X_{221}+X_{222}+X_{223}+X_{321}+X_{322}+X_{323}=30 ;$

$X_{131}+X_{132}+X_{133}+X_{231}+X_{232}+X_{233}+X_{331}+X_{332}+X_{333}=30$;

$-8.18 Y_{111}+X_{111} \leq 0 ;-13.57 Y_{112}+X_{112} \leq 0$;

$-73.16 Y_{113}+X_{113} \leq 0 ;-2.4 Y_{121}+X_{121} \leq 0$;

$-5.78 Y_{122}+X_{122} \leq 0 ;-73.16 Y_{123}+X_{123} \leq 0$;

$-1.7 Y_{131}+X_{131} \leq 0 ;-6.48 Y_{132}+X_{132} \leq 0$;

$-73.16 Y_{133}+X_{133} \leq 0 ;-21.75 Y_{211}+X_{211} \leq 0$;

$-21.16 Y_{212}+X_{212} \leq 0 ;-73.16 Y_{213}+X_{213} \leq 0$;

$-8.18 Y_{221}+X_{221} \leq 0 ;-34.73 Y_{222}+X_{222} \leq 0$;

$-73.16 Y_{223}+X_{223} \leq 0 ;-2.18 Y_{231}+X_{231} \leq 0$;

$-6 Y_{232}+X_{232} \leq 0 ;-73.16 Y_{233}+X_{233} \leq 0$;

$-2.4 Y_{311}+X_{311} \leq 0 ;-5.76 Y_{312}+X_{312} \leq 0$;

$-73.16 Y_{313}+X_{313} \leq 0 ;-0.7 Y_{321}+X_{321} \leq 0$;

$-7.48 Y_{322}+X_{322} \leq 0 ;-73.16 Y_{323}+X_{323} \leq 0$;

$-2.18 Y_{331}+X_{311} \leq 0 ;-6 Y_{332}+X_{332} \leq 0$;

$-73.16 Y_{333}+X_{333} \leq 0 ;-8.18 Y_{112}+X_{111} \geq 0$;

$-13.57 Y_{113}+X_{112} \geq 0 ;-2.4 Y_{122}+X_{121} \geq 0$;

$-5.78 Y_{123}+X_{122} \geq 0 ;-1.7 Y_{132}+X_{131} \geq 0$;

$-6.48 Y_{133}+X_{132} \geq 0 ;-21.75 Y_{212}+X_{211} \geq 0$;

$-21.16 Y_{213}+X_{212} \geq 0 ;-8.18 Y_{222}+X_{221} \geq 0$;

$-34.73 Y_{223}+X_{222} \geq 0 ;-2.18 Y_{232}+X_{231} \geq 0$;

$-6 Y_{233}+X_{232} \geq 0 ;-2.4 Y_{312}+X_{311} \geq 0$;

$-5.78 Y_{313}+X_{312} \geq 0 ;-0.7 Y_{322}+X_{321} \geq 0$;

$-7.48 Y_{323}+X_{322} \geq 0 ;-2.18 Y_{332}+X_{331} \geq 0$;

$-6 Y_{333}+X_{332} \geq 0$;

where $X_{i j k} \geq 0, Y_{i j k} \geq 0$ or $1 ; i=1,2,3 ; j=1,2,3 ; k=1,2,3$.

Case III. $(\lambda=0.75)$. Substituting $\lambda=0.75$ in the Value index in Table 4, we obtain the Value of the intuitionistic fuzzy cost and intuitionistic fuzzy price breakpoints, which is given in Table 7.
Model I: now, using the above Value of the intuitionistic fuzzy cost and intuitionistic fuzzy price breakpoints, the transportation problem with intuitionistic fuzzy quantity discounts is converted to the following linear programming problem: 


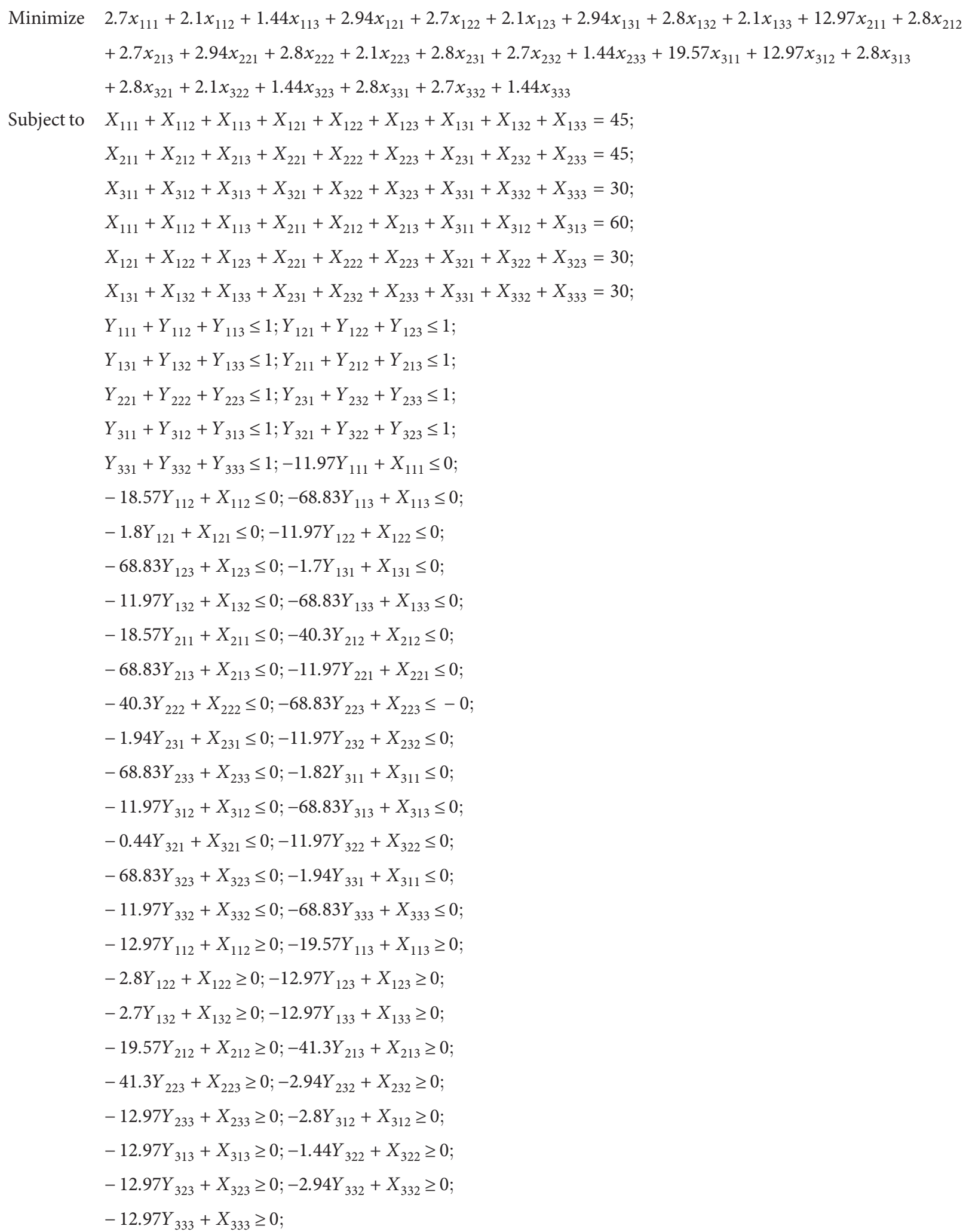

where $X_{i j k} \geq 0, Y_{i j k} \geq 0$ or $1 ; i=1,2,3 ; j=1,2,3 ; k=1,2,3$. Model II: similarly, using the Value of the intuitionistic fuzzy cost and intuitionistic fuzzy price breakpoints in
Table 6, the transportation problem with intuitionistic fuzzy incremental quantity discounts is converted to the following linear programming problem: 


$$
\begin{aligned}
& \text { Minimize } 2.7 x_{111}+2.1 x_{112}+1.44 x_{113}+2.94 x_{121}+2.7 x_{122}+2.1 x_{123}+2.94 x_{131}+2.8 x_{132}+2.1 x_{133}+12.97 x_{211} \\
& +12.97 x_{312}+2.8 x_{313}+2.8 x_{321}+2.1 x_{322}+1.44 x_{323}+2.8 x_{331}+2.7 x_{332}+1.44 x_{333} \\
& \text { Subject to } X_{111}+X_{112}+X_{113}+X_{121}+X_{122}+X_{123}+X_{131}+X_{132}+X_{133}=45 \text {; } \\
& X_{211}+X_{212}+X_{213}+X_{221}+X_{222}+X_{223}+X_{231}+X_{232}+X_{233}=45 \\
& X_{311}+X_{312}+X_{313}+X_{321}+X_{322}+X_{323}+X_{331}+X_{332}+X_{333}=30 \\
& X_{111}+X_{112}+X_{113}+X_{211}+X_{212}+X_{213}+X_{311}+X_{312}+X_{313}=60 \\
& X_{121}+X_{122}+X_{123}+X_{221}+X_{222}+X_{223}+X_{321}+X_{322}+X_{323}=30 \text {; } \\
& X_{131}+X_{132}+X_{133}+X_{231}+X_{232}+X_{233}+X_{331}+X_{332}+X_{333}=30 \text {; } \\
& -11.97 Y_{111}+X_{111} \leq 0 ;-6.6 Y_{112}+X_{112} \leq 0 \text {; } \\
& -68.83 Y_{113}+X_{113} \leq 0 ;-1.8 Y_{121}+X_{121} \leq 0 \text {; } \\
& -10.17 Y_{122}+X_{122} \leq 0 ;-68.83 Y_{123}+X_{123} \leq 0 \\
& -1.7 Y_{131}+X_{131} \leq 0 ;-10.27 Y_{132}+X_{132} \leq 0 \text {; } \\
& -68.83 Y_{133}+X_{133} \leq 0 ;-18.57 Y_{211}+X_{211} \leq 0 \\
& -21.73 Y_{212}+X_{212} \leq 0 ;-68.83 Y_{213}+X_{213} \leq 0 \\
& -11.97 Y_{221}+X_{221} \leq 0 ;-28.33 Y_{222}+X_{222} \leq 0 \text {; } \\
& -68.83 Y_{223}+X_{223} \leq 0 ;-1.94 Y_{231}+X_{231} \leq 0 \text {; } \\
& -10.03 Y_{232}+X_{232} \leq 0 ;-68.83 Y_{233}+X_{233} \leq 0 \text {; } \\
& -1.8 Y_{311}+X_{311} \leq 0 ;-10.17 Y_{312}+X_{312} \leq 0 \text {; } \\
& -68.83 Y_{313}+X_{313} \leq 0 ;-1.94 Y_{331}+X_{311} \leq 0 \text {; } \\
& -10.03 Y_{332}+X_{332} \leq 0 ;-68.83 Y_{333}+X_{333} \leq 0 \text {; } \\
& -11.97 Y_{112}+X_{111} \geq 0 ;-6.6 Y_{113}+X_{112} \geq 0 \text {; } \\
& -1.8 Y_{122}+X_{121} \geq 0 ;-10.11 Y_{123}+X_{122} \geq 0 \text {; } \\
& -1.7 Y_{132}+X_{131} \geq 0 ;-10.27 Y_{133}+X_{132} \geq 0 \text {; } \\
& -18.57 Y_{212}+X_{211} \geq 0 ;-21.73 Y_{213}+X_{212} \geq 0 \text {; } \\
& -11.9 Y_{222}+X_{221} \geq 0 ;-28.33 Y_{223}+X_{222} \geq 0 \text {; } \\
& -1.94 Y_{232}+X_{231} \geq 0 ;-10.03 Y_{233}+X_{232} \geq 0 \text {; } \\
& -1.8 Y_{312}+X_{311} \geq 0 ;-10.17 Y_{313}+X_{312} \geq 0 \text {; } \\
& -0.44 Y_{322}+X_{321} \geq 0 ;-11.53 Y_{323}+X_{322} \geq 0 \text {; } \\
& -1.94 Y_{332}+X_{331} \geq 0 ;-10.03 Y_{333}+X_{332} \geq 0 \text {; }
\end{aligned}
$$$$
+2.8 x_{212}+2.7 x_{213}+2.94 x_{221}+2.8 x_{222}+2.1 x_{223}+2.8 x_{231}+2.7 x_{232}+1.44 x_{233}+19.57 x_{311}
$$

where $X_{i j k} \geq 0, Y_{i j k} \geq 0$ or $1 ; i=1,2,3 ; j=1,2,3 ; k=1,2,3$.

4.4.2. Ambiguity-Based Intuitionistic Fuzzy Quantity Discount and Incremental Intuitionistic Fuzzy Quantity Discount Transportation Problems. In order to compute the Ambiguity of the solution, we formulate the Ambiguitybased intuitionistic fuzzy quantity discount and incremental intuitionistic fuzzy quantity discount transportation problems. In this regard, Ambiguity measure of all cost and price breakpoints is calculated. Then, the Ambiguity index is evaluated, which is shown in Table 8.

Case IV. I $(\lambda=0.25)$. Substituting $\lambda=0.25$ in the Ambiguity index in Table 8, the Ambiguity measures of the intuitionistic fuzzy cost and intuitionistic fuzzy price breakpoints are provided in Table 9. 
TABLE 8: Ambiguity of the IF cost and IF price breakpoints.

$\widetilde{q}_{11 k}: \widetilde{C}_{11 k}, k=1,2,3$

$0.27 \lambda+(1-\lambda) 0.47 \leq X_{111}<0.6 \lambda+(1-\lambda) 0.98: 0.3 \lambda+(1-\lambda) 0.65$

$0.6 \lambda+(1-\lambda) 0.98 \leq X_{112}<0.3 \lambda+(1-\lambda) 0.75: 0.41 \lambda+(1-\lambda) 0.57$

$0.3 \lambda+(1-\lambda) 0.75 \leq X_{113}<0.49 \lambda+(1-\lambda) 0.85: 0.42 \lambda+(1-\lambda) 0.82$

$\widetilde{q}_{12 k}: \widetilde{C}_{12 k}, k=1,2,3$

$0.27 \lambda+(1-\lambda) 0.47 \leq X_{121}<0.36 \lambda+(1-\lambda) 0.65: 0.36 \lambda+(1-\lambda)$

0.82

$0.36 \lambda+(1-\lambda) 0.65 \leq X_{212}<0.6 \lambda+(1-\lambda) 0.98: 0.3 \lambda+(1-\lambda) 0.65$

$0.6 \lambda+(1-\lambda) 0.98 \leq X_{123}<0.49 \lambda+(1-\lambda) 0.85: 0.41 \lambda+(1-\lambda)$

0.57

$\widetilde{q}_{13 k}: \widetilde{C}_{13 k}, k=1,2,3$

$0.27 \lambda+(1-\lambda) 0.47 \leq X_{121}<0.41 \lambda+(1-\lambda) 0.57: 0.36 \lambda+(1-\lambda)$

0.65

$0.41 \lambda+(1-\lambda) 0.57 \leq X_{112}<0.6 \lambda+(1-\lambda) 0.98: 0.36 \lambda+(1-\lambda) 0.82$

$0.6 \lambda+(1-\lambda) 0.98 \leq X_{123}<0.49 \lambda+(1-\lambda) 0.85: 0.3 \lambda+(1-\lambda) 0.65$

$\widetilde{q}_{21 k}: \widetilde{C}_{21 k}, k=1,2,3$

$0.27 \lambda+(1-\lambda) 0.47 \leq X_{211}<0.3 \lambda+(1-\lambda) 0.75: 0.6 \lambda+(1-\lambda) 0.98$

$0.3 \lambda+(1-\lambda) 0.75 \leq X_{212}<0.36 \lambda+(1-\lambda) 0.65: 0.36 \lambda+(1-\lambda)$

0.65

$0.36 \lambda+(1-\lambda) 0.65 \leq X_{213}<0.49 \lambda+(1-\lambda) 0.85: 0.3 \lambda+(1-\lambda)$

0.74

$\tilde{q}_{22 k}: \widetilde{C}_{22 k}, k=1,2,3$

$0.27 \lambda+(1-\lambda) 0.47 \leq X_{221}<0.6 \lambda+(1-\lambda) 0.98: 0.36 \lambda+(1-\lambda)$

0.65

$0.6 \lambda+(1-\lambda) 0.98 \leq X_{222}<0.36 \lambda+(1-\lambda) 0.65: 0.36 \lambda+(1-\lambda)$

0.82

$0.36 \lambda+(1-\lambda) 0.65 \leq X_{2123}<0.49 \lambda+(1-\lambda) 0.85: 0.3 \lambda+(1-\lambda)$ 0.65

$\widetilde{q}_{23 k}: \widetilde{C}_{23 k}, k=1,2,3$

$0.27 \lambda+(1-\lambda) 0.47 \leq X_{231}<0.36 \lambda+(1-\lambda) 0.82: 0.36 \lambda+(1-\lambda)$

0.65

$0.36 \lambda+(1-\lambda) 0.82 \leq X_{232}<0.6 \lambda+(1-\lambda) 0.98: 0.3 \lambda+(1-\lambda) 0.74$

$0.6 \lambda+(1-\lambda) 0.98 \leq X_{233}<0.49 \lambda+(1-\lambda) 0.85: 0.42 \lambda+(1-\lambda)$

0.82

$\widetilde{q}_{31 k}: \widetilde{C}_{31 k}, k=1,2,3$

$0.27 \lambda+(1-\lambda) 0.47 \leq X_{311}<0.36 \lambda+(1-\lambda) 0.65: 0.3 \lambda+(1-\lambda)$

0.75

$0.36 \lambda+(1-\lambda) 0.65 \leq X_{312}<0.6 \lambda+(1-\lambda) 0.98: 0.6 \lambda+(1-\lambda) 0.98$

$0.6 \lambda+(1-\lambda) 0.98 \leq X_{312}<0.49 \lambda+(1-\lambda) 0.85: 0.36 \lambda+(1-\lambda)$

0.65

$\widetilde{q}_{32 k}: \widetilde{C}_{32 k}, k=1,2,3$

$0.27 \lambda+(1-\lambda) 0.47 \leq X_{321}<0.42 \lambda+(1-\lambda) 0.82: 0.36 \lambda+(1-\lambda)$

0.65

$0.42 \lambda+(1-\lambda) 0.82 \leq X_{322}<0.6 \lambda+(1-\lambda) 0.98: 0.3 \lambda+(1-\lambda) 0.65$

$0.6 \lambda+(1-\lambda) 0.98 \leq X_{323}<0.49 \lambda+(1-\lambda) 0.85: 0.42 \lambda+(1-\lambda)$

0.82

$\widetilde{q}_{33 k}: \widetilde{C}_{33 k}, k=1,2,3$

$0.27 \lambda+(1-\lambda) 0.47 \leq X_{331}<0.3 \lambda+(1-\lambda) 0.74: 0.36 \lambda+(1-\lambda)$

0.65

$0.3 \lambda+(1-\lambda) 0.74 \leq X_{332}<0.3 \lambda+(1-\lambda) 0.75: 0.3 \lambda+(1-\lambda) 0.74$

$0.3 \lambda+(1-\lambda) 0.75 \leq X_{333}<0.49 \lambda+(1-\lambda) 0.85: 0.42 \lambda+(1-\lambda)$

0.82

Model I: Now, using the Ambiguity measures of the intuitionistic fuzzy cost and intuitionistic fuzzy price breakpoints provided in Table 9, the transportation problem with intuitionistic fuzzy quantity discounts is converted to the following linear programming problem:
Minimize $\quad 0.48 x_{111}+0.46 x_{112}+0.44 x_{113}+0.5 x_{121}$ $+0.48 x_{122}+0.46 x_{123}+0.53 x_{131}+0.5 x_{132}$ $+0.48 x_{133}+0.54 x_{211}+0.53 x_{212}+0.46 x_{213}$ $+0.53 x_{221}+0.5 x_{222}+0.48 x_{223}+0.53 x_{231}$ $+0.56 x_{232}+0.44 x_{233}+0.46 x_{311}+0.45 x_{312}$ $+0.53 x_{313}+0.53 x_{321}+0.48 x_{322}+0.44 x_{323}$ $+0.53 x_{331}+0.46 x_{332}+0.44 x_{333}$

Subject to $X_{111}+X_{112}+X_{113}+X_{121}+X_{122}+X_{123}+X_{131}$ $+X_{132}+X_{133}=45$

$X_{211}+X_{212}+X_{213}+X_{221}+X_{222}+X_{223}+X_{231}$ $+X_{232}+X_{233}=45$

$X_{311}+X_{312}+X_{313}+X_{321}+X_{322}+X_{323}+X_{331}$ $+X_{332}+X_{333}=30$

$X_{111}+X_{112}+X_{113}+X_{211}+X_{212}+X_{213}+X_{311}$ $+X_{312}+X_{313}=60$

$X_{121}+X_{122}+X_{123}+X_{221}+X_{222}+X_{223}+X_{321}$ $+X_{322}+X_{323}=30$

$X_{131}+X_{132}+X_{133}+X_{231}+X_{232}+X_{233}+X_{331}$ $+X_{332}+X_{333}=30$

$Y_{111}+Y_{112}+Y_{113} \leq 1 ; Y_{121}+Y_{122}+Y_{123} \leq 1 ;$

$Y_{131}+Y_{132}+Y_{133} \leq 1 ; Y_{211}+Y_{212}+Y_{213} \leq 1 ;$

$Y_{221}+Y_{222}+Y_{223} \leq 1 ; Y_{231}+Y_{232}+Y_{233} \leq 1 ;$

$Y_{311}+Y_{312}+Y_{313} \leq 1 ; Y_{321}+Y_{322}+Y_{323} \leq 1 ;$

$Y_{331}+Y_{332}+Y_{333} \leq 1 ;-0.46 Y_{111}+X_{111} \leq 0$;

$-0.36 Y_{112}+X_{112} \leq 0 ;-1.45 Y_{113}+X_{113} \leq 0$;

$-0.47 Y_{121}+X_{121} \leq 0 ;-0.46 Y_{122}+X_{122} \leq 0$;

$-1.45 Y_{123}+X_{123} \leq 0 ;-0.54 Y_{131}+X_{131} \leq 0$;

$-0.46 Y_{132}+X_{132} \leq 0 ;-1.45 Y_{133}+X_{133} \leq 0$;

$-0.36 Y_{211}+X_{211} \leq 0 ;-0.15 Y_{212}+X_{212} \leq 0$;

$-1.45 Y_{213}+X_{213} \leq 0 ;-0.46 Y_{221}+X_{221} \leq 0$;

$-0.15 Y_{222}+X_{222} \leq 0 ;-1.45 Y_{223}+X_{223} \leq 0$;

$-0.5 Y_{231}+X_{231} \leq 0 ;-0.46 Y_{232}+X_{232} \leq 0$;

$-1.45 Y_{233}+X_{233} \leq 0 ;-0.47 Y_{311}+X_{311} \leq 0$;

$-0.46 Y_{312}+X_{312} \leq 0 ;-1.45 Y_{313}+X_{313} \leq 0$;

$-0.56 Y_{321}+X_{321} \leq 0 ;-0.46 Y_{322}+X_{322} \leq 0$;

$-1.45 Y_{323}+X_{323} \leq 0 ;-0.5 Y_{331}+X_{311} \leq 0$;

$-0.46 Y_{332}+X_{332} \leq 0 ;-1.45 Y_{333}+X_{333} \leq 0$;

$-0.54 Y_{112}+X_{112} \geq 0 ;-0.64 Y_{113}+X_{113} \geq 0 ;$

$-0.53 Y_{122}+X_{122} \geq 0 ;-0.54 Y_{123}+X_{123} \geq 0$;

$-0.46 Y_{132}+X_{132} \geq 0 ;-0.54 Y_{133}+X_{133} \geq 0$;

$-0.64 Y_{212}+X_{212} \geq 0 ;-0.85 Y_{213}+X_{213} \geq 0$;

$-0.54 Y_{222}+X_{222} \geq 0 ;-0.85 Y_{223}+X_{223} \geq 0$;

$-0.5 Y_{232}+X_{232} \geq 0 ;-0.54 Y_{233}+X_{233} \geq 0$;

$-0.53 Y_{312}+X_{312} \geq 0 ;-0.54 Y_{313}+X_{313} \geq 0$;

$-0.44 Y_{322}+X_{322} \geq 0 ;-0.54 Y_{323}+X_{323} \geq 0$;

$-0.5 Y_{332}+X_{332} \geq 0$;

$-0.54 Y_{333}+X_{333} \geq 0$ 
TABLE 9: Ambiguity measures of the IF cost and IF price breakpoints when $\lambda=0.25$.

\begin{tabular}{cccc}
\hline & $\lambda=0.25$ & \\
\hline & & & \\
& $0.41 \leq X_{111}<0.54: 0.48$ & $0.41 \leq X_{121}<0.53: 0.5$ & $0.41 \leq X_{131}<0.46: 0.53$ \\
& $0.54 \leq X_{112}<0.64: 0.46$ & $0.53=X_{122}<0.54: 0.48$ & $0.46 \leq X_{132}<0.54: 0.5$ \\
Demand & $0.64=X_{113}<1.45: 0.44$ & $0.54=X_{123}<1.45: 0.46$ & $0.54=X_{133}<1.45: 0.48$ \\
& $0.41=X_{211}<0.64: 0.54$ & $0.41=X_{221}<0.54: 0.53$ & $0.41=X_{231}<0.5: 0.53$ \\
& $0.64=X_{212}<0.85: 0.53$ & $0.54=X_{222}<0.85: 0.5$ & $0.5=X_{232}<0.54: 0.46$ \\
& $0.85=X_{213}<1.45: 0.46$ & $0.85=X_{223}<1.45: 0.48$ & $0.54=X_{233}<1.45: 0.44$ \\
& $0.41=X_{311}<0.53: 0.64$ & $0.41=X_{321}<0.44: 0.53$ & $0.41=X_{331}<0.5: 0.53$ \\
$0.53=X_{312}<0.54: 0.54$ & $0.44=X_{322}<0.54: 0.48$ & $0.5=X_{332}<0.54: 0.46$ \\
$0.54=X_{313}<1.45: 0.53$ & $0.54=X_{323}<1.45: 0.44$ & $0.54=X_{333}<1.45: 0.44$ & 30 \\
60 & 30 & 30 \\
\hline
\end{tabular}

where $X_{i j k} \geq 0, Y_{i j k} \geq 0$ or $1 ; i=1,2,3 ; j=1,2,3 ; k=1,2,3$.

Model II: similarly, using the Value of the intuitionistic fuzzy cost and intuitionistic fuzzy price breakpoints in Table 9, the transportation problem with intuitionistic fuzzy incremental quantity discounts is converted to the following linear programming problem:

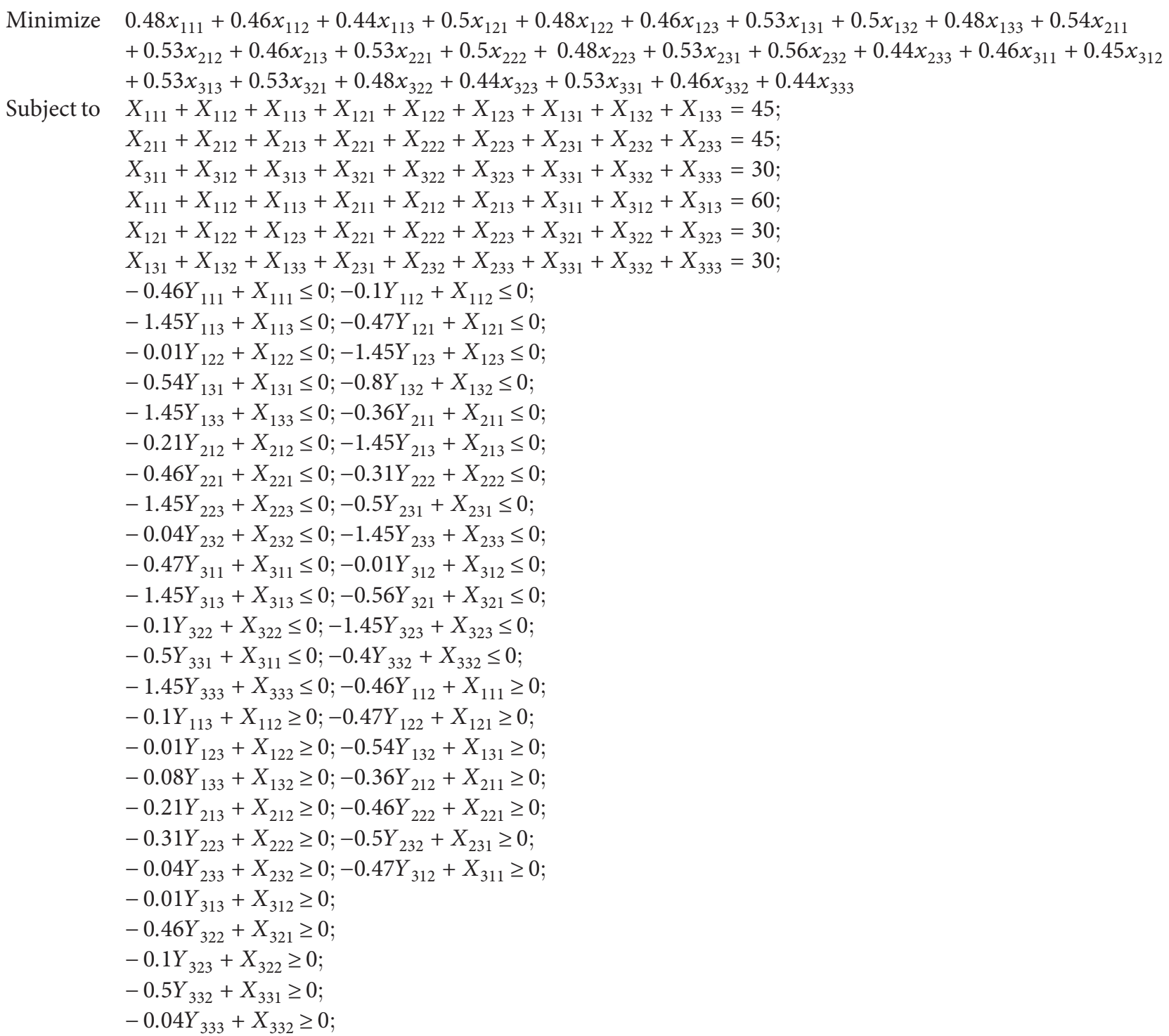


where $X_{i j k} \geq 0, Y_{i j k} \geq 0$ or $1 ; i=1,2,3 ; j=1,2,3 ; k=1,2$, 3.

Case V. $\operatorname{II}(\lambda=0.50)$. Substituting $\lambda=0.50$ in the Ambiguity index in Table 8, the Ambiguity measures of the intuitionistic fuzzy cost and intuitionistic fuzzy price breakpoints are provided in Table 10.

From the ambiguity measures of the intuitionistic fuzzy cost and intuitionistic fuzzy price breakpoints in Table 10, the transportation problem with intuitionistic fuzzy quantity discounts and intuitionistic fuzzy incremental quantity discounts is transformed into the linear programming problems Model I and Model II, respectively.

Model I: now, using the Ambiguity measures of the intuitionistic fuzzy cost and intuitionistic fuzzy price breakpoints provided in Table 10, the transportation problem with intuitionistic fuzzy quantity discounts is converted to the following linear programming problem:

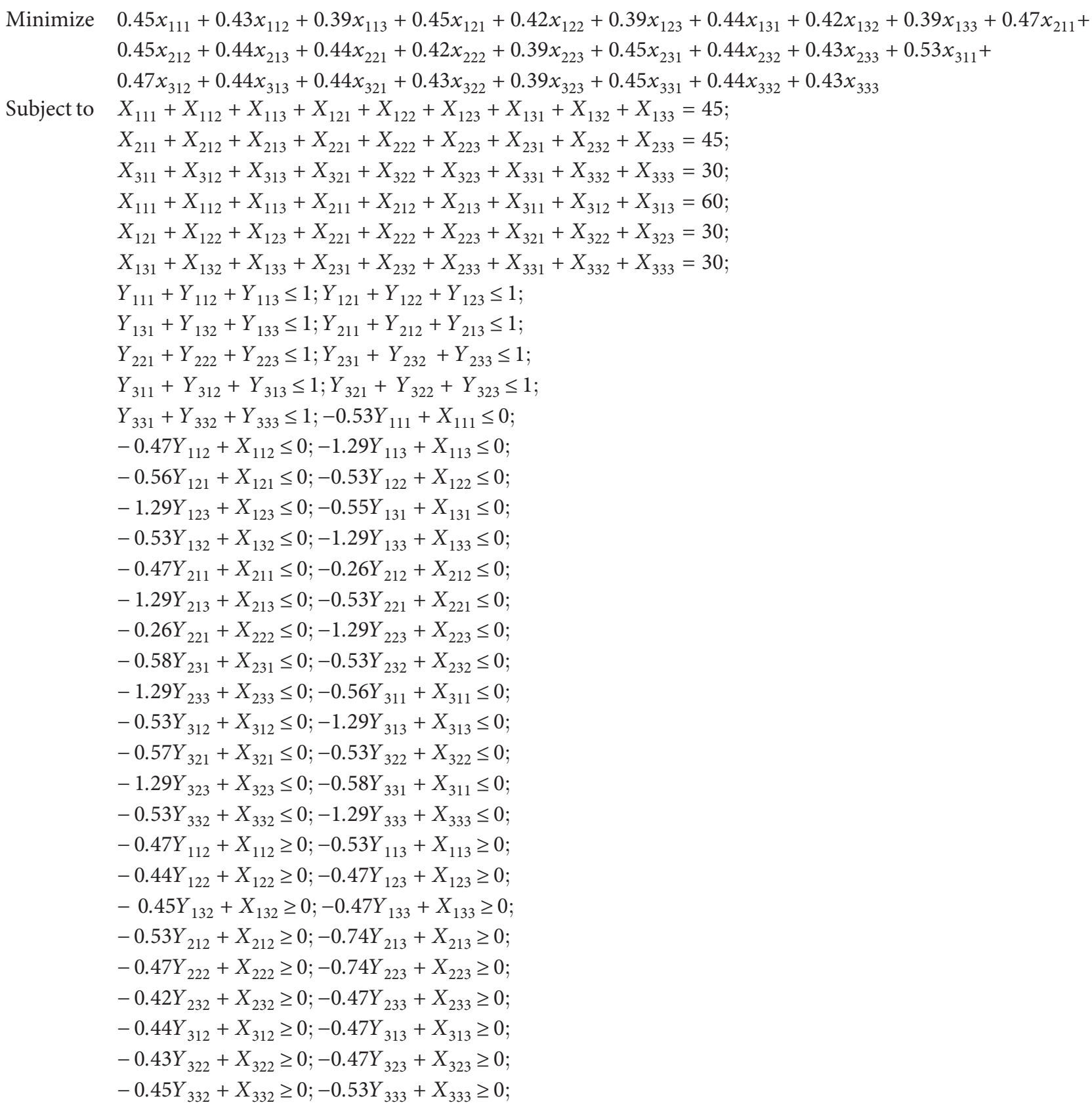


TABLE 10: Ambiguity of the IF cost and IF price breakpoints when $\lambda=0.50$.

\begin{tabular}{|c|c|c|c|c|}
\hline & & $=0.50$ & & Supply \\
\hline \multirow{10}{*}{ Demand } & $0.34 \leq X_{111}<0.47: 0.45$ & $0.34 \leq X_{121}<0.44: 0.45$ & $0.34 \leq X_{131}<0.45: 0.44$ & \multirow{3}{*}{45} \\
\hline & $0.47 \leq X_{112}<0.53: 0.43$ & $0.44=X_{122}<0.47: 0.42$ & $0.45 \leq X_{132}<0.47: 0.42$ & \\
\hline & $0.53=X_{113}<1.29: 0.39$ & $0.47=X_{123}<1.29: 0.39$ & $0.47=X_{133}<1.29: 0.39$ & \\
\hline & $0.34=X_{211}<0.53: 0.47$ & $0.34=X_{221}<0.47: 0.44$ & $0.34=X_{231}<0.42: 0.45$ & \multirow{3}{*}{45} \\
\hline & $0.53=X_{212}<0.74: 0.45$ & $0.47=X_{222}<0.74: 0.42$ & $0.42=X_{232}<0.47: 0.44$ & \\
\hline & $0.74=X_{213}<1.450 .44$ & $0.74=X_{223}<1.29: 0.39$ & $0.47=X_{233}<1.29: 0.43$ & \\
\hline & $0.34=X_{311}<0.44: 0.53$ & $0.34=X_{321}<0.43: 0.44$ & $0.34=X_{331}<0.42: 0.45$ & \multirow{3}{*}{30} \\
\hline & $0.44=X_{312}<0.47: 0.47$ & $0.43=X_{322}<0.47: 0.43$ & $0.42=X_{332}<0.47: 0.44$ & \\
\hline & $0.47=X_{313}<1.45: 0.44$ & $0.47=X_{323}<1.29: 0.39$ & $0.47=X_{333}<1.29: 0.43$ & \\
\hline & 60 & 30 & 30 & 120 \\
\hline
\end{tabular}

where $X_{i j k} \geq 0, Y_{i j k} \geq 0$ or $1 ; i=1,2,3, j=1,2,3 ; k=1,2,3$. Model II: similarly, using the Value of the intuitionistic fuzzy cost and intuitionistic fuzzy price breakpoints in
Table 10, the transportation problem with intuitionistic fuzzy incremental quantity discounts is converted to the following linear programming problem:
Minimize

$0.45 x_{111}+0.43 x_{112}+0.39 x_{113}+0.45 x_{121}+0.42 x_{122}+0.39 x_{123}+0.44 x_{131}+0.42 x_{132}+0.39 x_{133}$

$+0.47 x_{211}+0.45 x_{212}+0.44 x_{213}+0.44 x_{221}+0.42 x_{222}+0.39 x_{223}+0.45 x_{231}+0.44 x_{232}+0.43 x_{233}+0.53 x_{311}$

$+0.47 x_{312}+0.44 x_{313}+0.44 x_{321}+0.43 x_{322}+0.39 x_{323}+0.45 x_{331}+0.44 x_{332}+0.43 x_{333}$

Subject to $X_{111}+X_{112}+X_{113}+X_{121}+X_{122}+X_{123}+X_{131}+X_{132}+X_{133}=45$

$X_{211}+X_{212}+X_{213}+X_{221}+X_{222}+X_{223}+X_{231}+X_{232}+X_{233}=45$

$X_{311}+X_{312}+X_{313}+X_{321}+X_{322}+X_{323}+X_{331}+X_{332}+X_{333}=30 ;$

$X_{111}+X_{112}+X_{113}+X_{211}+X_{212}+X_{213}+X_{311}+X_{312}+X_{313}=60 ;$

$X_{121}+X_{122}+X_{123}+X_{221}+X_{222}+X_{223}+X_{321}+X_{322}+X_{323}=30 ;$

$X_{131}+X_{132}+X_{133}+X_{231}+X_{232}+X_{233}+X_{331}+X_{332}+X_{333}=30$;

$-0.53 Y_{111}+X_{111} \leq 0 ;-0.06 Y_{112}+X_{112} \leq 0$;

$-1.29 Y_{113}+X_{113} \leq 0 ;-0.56 Y_{121}+X_{121} \leq 0$;

$-0.03 Y_{122}+X_{122} \leq 0 ;-1.29 Y_{123}+X_{123} \leq 0$;

$-0.55 Y_{131}+X_{131} \leq 0 ;-0.02 Y_{132}+X_{132} \leq 0$;

$-1.29 Y_{133}+X_{133} \leq 0 ;-0.47 Y_{211}+X_{211} \leq 0$;

$-0.21 Y_{212}+X_{212} \leq 0 ;-1.29 Y_{213}+X_{213} \leq 0$;

$-0.53 Y_{221}+X_{221} \leq 0 ;-0.27 Y_{222}+X_{222} \leq 0$;

$-1.29 Y_{223}+X_{223} \leq 0 ;-0.58 Y_{231}+X_{231} \leq 0$;

$-0.05 Y_{232}+X_{232} \leq 0 ;-1.29 Y_{233}+X_{233} \leq 0$;

$-0.56 Y_{311}+X_{311} \leq 0 ;-0.03 Y_{312}+X_{312} \leq 0$;

$-1.29 Y_{313}+X_{313} \leq 0 ;-0.57 Y_{321}+X_{321} \leq 0$;

$-0.4 Y_{322}+X_{322} \leq 0 ;-1.29 Y_{323}+X_{323} \leq 0$;

$-0.58 Y_{331}+X_{311} \leq 0 ;-0.05 Y_{332}+X_{332} \leq 0$;

$-1.29 Y_{333}+X_{333} \leq 0 ;-0.53 Y_{112}+X_{111} \geq 0$;

$-0.06 Y_{113}+X_{112} \geq 0 ;-0.56 Y_{122}+X_{121} \geq 0$;

$-0.03 Y_{123}+X_{122} \geq 0 ;-0.55 Y_{132}+X_{131} \geq 0$;

$-0.02 Y_{133}+X_{132} \geq 0 ;-0.47 Y_{212}+X_{211} \geq 0$;

$-0.21 Y_{213}+X_{212} \geq 0 ;-0.53 Y_{222}+X_{221} \geq 0$;

$-0.31 Y_{223}+X_{222} \geq 0 ;-0.58 Y_{232}+X_{231} \geq 0$;

$-0.05 Y_{233}+X_{232} \geq 0 ;-0.56 Y_{312}+X_{311} \geq 0$;

$-0.03 Y_{313}+X_{312} \geq 0 ;-0.57 Y_{322}+X_{321} \geq 0$;

$-0.04 Y_{323}+X_{322} \geq 0 ;-0.58 Y_{332}+X_{331} \geq 0$;

$-0.05 Y_{333}+X_{332} \geq 0$; 
where $X_{i j k} \geq 0, Y_{i j k} \geq 0$ or $1 ; i=1,2,3 ; j=1,2,3 ; k=1,2$, 3.

Case VI. $\operatorname{III}(\lambda=0.75)$. Substituting $\lambda=0.75$ in the Ambiguity index in Table 8, the Ambiguity measures of the intuitionistic fuzzy cost and intuitionistic fuzzy price breakpoints are provided in Table 11.

From the Ambiguity measures of the intuitionistic fuzzy cost and intuitionistic fuzzy price breakpoints in Table 11, the transportation problem with intuitionistic fuzzy quantity discounts and intuitionistic fuzzy incremental quantity discounts is transformed into the linear programming problems Model I and Model II, respectively.

Model I: now, using the Ambiguity measures of the intuitionistic fuzzy cost and intuitionistic fuzzy price breakpoints provided in Table 11, the transportation problem with intuitionistic fuzzy quantity discounts is converted to the following linear programming problem:

$$
\begin{aligned}
& \text { Minimize } \quad 0.45 x_{111}+0.35 x_{112}+0.29 x_{113}+0.45 x_{121}+0.37 x_{122}+0.29 x_{123}+0.39 x_{131}+0.37 x_{132}+0.29 x_{133}+0.45 x_{211}+ \\
& 0.41 x_{212}+0.39 x_{213}+0.39 x_{221}+0.37 x_{222}+0.29 x_{223}+0.45 x_{231}+0.39 x_{232}+0.35 x_{233}+0.44 x_{311}+ \\
& 0.41 x_{312}+0.39 x_{313}+0.39 x_{321}+0.35 x_{322}+0.29 x_{323}+0.45 x_{331}+0.39 x_{332}+0.35 x_{333} \\
& \text { Subject to } X_{111}+X_{112}+X_{113}+X_{121}+X_{122}+X_{123}+X_{131}+X_{132}+X_{133}=45 \\
& X_{211}+X_{212}+X_{213}+X_{221}+X_{222}+X_{223}+X_{231}+X_{232}+X_{233}=45 \\
& X_{311}+X_{312}+X_{313}+X_{321}+X_{322}+X_{323}+X_{331}+X_{332}+X_{333}=30 ; \\
& X_{111}+X_{112}+X_{113}+X_{211}+X_{212}+X_{213}+X_{311}+X_{312}+X_{313}=60 ; \\
& X_{121}+X_{122}+X_{123}+X_{221}+X_{222}+X_{223}+X_{321}+X_{322}+X_{323}=30 \text {; } \\
& X_{131}+X_{132}+X_{133}+X_{231}+X_{232}+X_{233}+X_{331}+X_{332}+X_{333}=30 \\
& Y_{111}+Y_{112}+Y_{113} \leq 1 ; Y_{121}+Y_{122}+Y_{123} \leq 1 ; \\
& Y_{131}+Y_{132}+Y_{133} \leq 1 ; Y_{211}+Y_{212}+Y_{213} \leq 1 ; \\
& Y_{221}+Y_{222}+Y_{223} \leq 1 ; Y_{231}+Y_{232}+Y_{233} \leq 1 ; \\
& Y_{311}+Y_{312}+Y_{313} \leq 1 ; Y_{321}+Y_{322}+Y_{323} \leq 1 ; \\
& Y_{331}+Y_{332}+Y_{333} \leq 1 ;-0.59 Y_{111}+X_{111} \leq 0 \text {; } \\
& -0.56 Y_{112}+X_{112} \leq 0 ;-1.13 Y_{113}+X_{113} \leq 0 \text {; } \\
& -0.61 Y_{121}+X_{121} \leq 0 ;-0.59 Y_{122}+X_{122} \leq 0 \text {; } \\
& -1.13 Y_{123}+X_{123} \leq 0 ;-0.59 Y_{131}+X_{131} \leq 0 \text {; } \\
& -0.55 Y_{132}+X_{132} \leq 0 ;-1.13 Y_{133}+X_{133} \leq 0 \text {; } \\
& -0.56 Y_{211}+X_{211} \leq 0 ;-0.36 Y_{212}+X_{212} \leq 0 \text {; } \\
& -1.13 Y_{213}+X_{213} \leq 0 ;-0.59 Y_{221}+X_{221} \leq 0 \text {; } \\
& -0.36 Y_{222}+X_{222} \leq 0 ;-1.13 Y_{223}+X_{223} \leq 0 \text {; } \\
& -0.63 Y_{231}+X_{231} \leq 0 ;-0.59 Y_{232}+X_{232} \leq 0 \text {; } \\
& -1.13 Y_{233}+X_{233} \leq 0 ;-0.61 Y_{311}+X_{311} \leq 0 \text {; } \\
& -0.59 Y_{312}+X_{312} \leq 0 ;-1.13 Y_{313}+X_{313} \leq 0 \text {; } \\
& -0.65 Y_{321}+X_{321} \leq 0 ;-0.59 Y_{322}+X_{322} \leq 0 \text {; } \\
& -1.13 Y_{323}+X_{323} \leq 0 ;-0.63 Y_{331}+X_{311} \leq 0 \text {; } \\
& -0.59 Y_{332}+X_{332} \leq 0 ;-1.13 Y_{333}+X_{333} \leq 0 \text {; } \\
& -0.41 Y_{112}+X_{112} \geq 0 ;-0.44 Y_{113}+X_{113} \geq 0 \text {; } \\
& -0.39 Y_{122}+X_{122} \geq 0 ;-0.41 Y_{123}+X_{123} \geq 0 \text {; } \\
& -0.41 Y_{132}+X_{132} \geq 0 ;-0.05 Y_{133}+X_{133} \geq 0 \text {; } \\
& -0.44 Y_{212}+X_{212} \geq 0 ;-0.64 Y_{213}+X_{213} \geq 0 \text {; } \\
& -0.41 Y_{222}+X_{222} \geq 0 ;-0.64 Y_{223}+X_{223} \geq 0 \text {; } \\
& -0.37 Y_{232}+X_{232} \geq 0 ;-0.41 Y_{233}+X_{233} \geq 0 \text {; } \\
& -0.39 Y_{312}+X_{312} \geq 0 ;-0.41 Y_{313}+X_{313} \geq 0 \text {; } \\
& -0.35 Y_{322}+X_{322} \geq 0 ;-0.41 Y_{323}+X_{323} \geq 0 \text {; } \\
& -0.37 Y_{332}+X_{332} \geq 0 \text {; } \\
& -0.41 Y_{333}+X_{333} \geq 0 \text {; }
\end{aligned}
$$


TABLE 11: Ambiguity measures of the IF cost and IF price breakpoints when $\lambda=0.75$.

\begin{tabular}{cccl}
\hline & $\lambda=0.75$ & \\
\hline $0.26 \leq X_{111}<0.41: 0.45$ & $0.26 \leq X_{121}<0.39: 0.45$ & $0.26 \leq X_{131}<0.41: 0.39$ \\
$0.41 \leq X_{112}<0.44: 0.35$ & $0.39=X_{122}<0.41: 0.37$ & $0.41 \leq X_{132}<0.45: 0.37$ \\
$0.44=X_{113}<1.13: 0.29$ & $0.41=X_{123}<1.13: 0.29$ & $0.45=X_{133}<1.13: 0.29$ \\
Demand & $0.26=X_{211}<0.44: 0.45$ & $0.26=X_{221}<0.41: 0.39$ & $0.26=X_{231}<0.37: 0.45$ \\
& $0.44=X_{212}<0.64: 0.41$ & $0.41=X_{222}<0.64: 0.37$ & $0.37=X_{232}<0.41: 0.39$ \\
& $0.64=X_{213}<1.13: 0.39$ & $0.64=X_{223}<1.13: 0.29$ & $0.41=X_{233}<1.13: 0.35$ \\
& $0.26=X_{311}<0.39: 0.44$ & $0.26=X_{321}<0.35: 0.39$ & $0.26=X_{331}<0.37: 0.45$ \\
& $0.39=X_{312}<0.41: 0.41$ & $0.35=X_{322}<0.41: 0.35$ & $0.37=X_{332}<0.41: 0.39$ \\
$0.41=X_{313}<1.13: 0.39$ & $0.41=X_{323}<1.13: 0.29$ & $0.41=X_{333}<1.13: 0.47$ \\
60 & 30 & 30 \\
\hline
\end{tabular}

where $X_{i j k} \geq 0, Y_{i j k} \geq 0$ or $1 ; i=1,2,3 ; j=1,2,3 ; k=1,2,3$. Model II: similarly, using the Value of the intuitionistic fuzzy cost and intuitionistic fuzzy price breakpoints in Table 11, the transportation problem with intuitionistic fuzzy incremental quantity discounts is converted to the following linear programming problem:

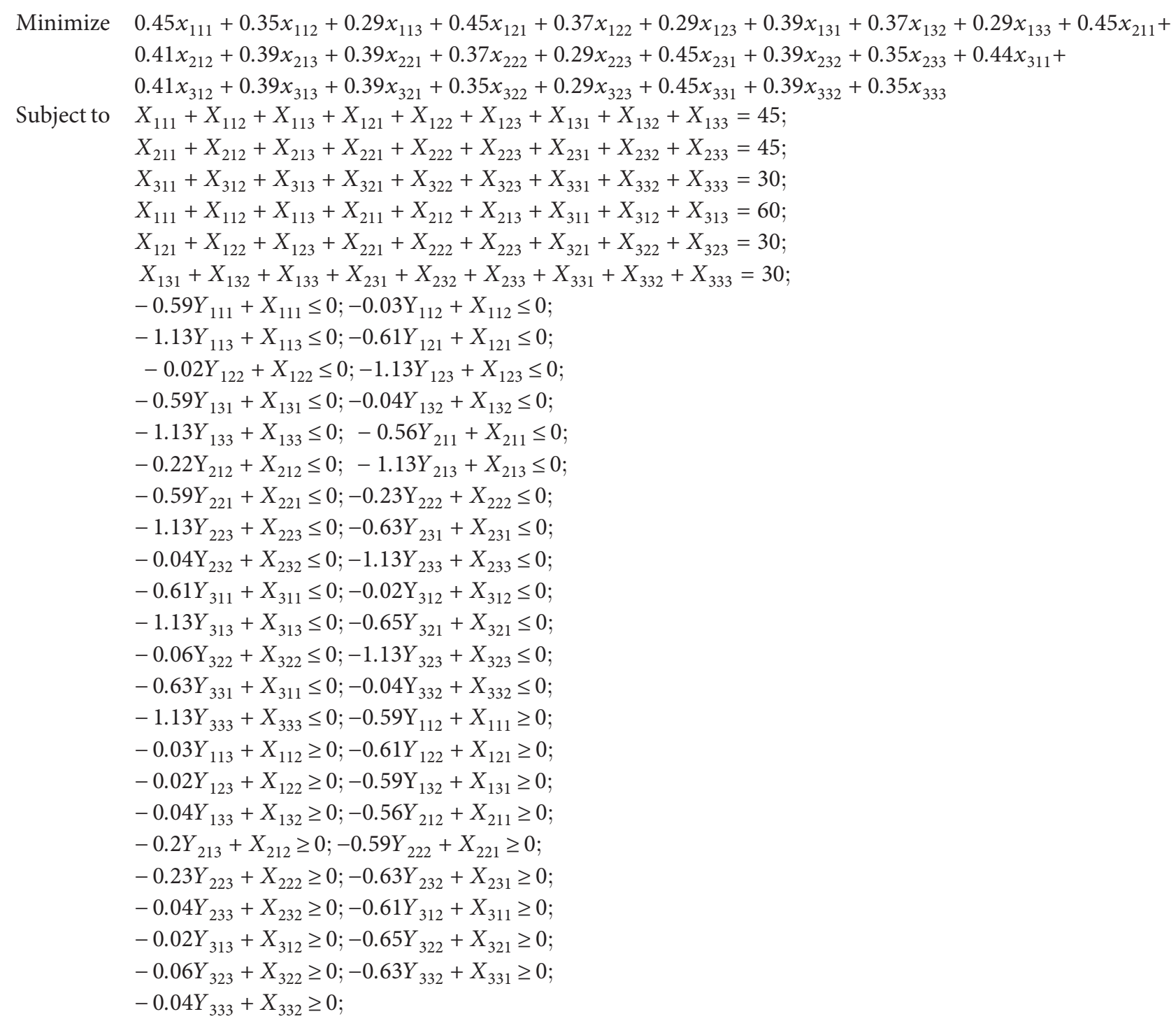


where $X_{i j k} \geq 0, Y_{i j k} \geq 0$ or $1 ; i=1,2,3 ; j=1,2,3 ; k=1,2$.

\section{Advantages and Limitations}

The transport cost is one of the most important influences in the pricing system. The cost of goods per unit transported from the source to the destination is considered to be fixed irrespective of the number of units transported. In fact, the cost of transport cannot be fixed exactly. In reality, volume discounts are often available for large shipments so that marginal shipping costs for one unit may follow a specific pattern. The main advantage of the proposed paper is to develop the mathematical model with a transportation problem with a quantity discount. Moreover, in the case of traditional transportation problems, it is believed that the decision-maker is certain of the exact values of the transportation costs, supply, and demand for the commodity. In real-world applications, due to uncontrollable variables, all these transport parameters cannot be determined precisely. Hence, here, the transport cost and price break quantities are represented by trapezoidal intuitionistic fuzzy numbers. Therefore, the proposed model is more realistic. Furthermore, most of the intuitionistic optimization problems are converted to linear problems using the ranking function. In the proposed paper, without using the ranking function, the problems are solved. The major drawback is when an intuitionistic fuzzy transportation problem is converted to a parametric transportation problem based on their Value indices and Ambiguity indices, the problems become more complex and have a high number of constraints.

\section{Results and Discussion}

Solving the above Value- and Ambiguity-based linear programming problems by using classical methods, we obtain the solution of the Value and Ambiguity for both Model I and Model II, which is provided in Tables 12 and 13.

(1) Value index given in Table 12 helps the buyer how to choose the best discount policy in TPIFQD and TPIIFQD.

(2) In the Value index TPIFQD, for the parameter Value 0.25 , the optimal Value is Rs. 261 and, for 0.5, the optimal Value is 219. For the parameter Value 0.75, the optimal Value is 191.7. As the parameter Value increased, the optimal Value decreased.

(3) In the Value index TPIIFQD also, as the parameter Value increased $(\lambda=0.25,0.5$, and 0.7$)$, the optimal Value decreased (319.72, 256.88, and 236.98).

(4) Here, the TPIFQD Value is less than the TPIIFQD value for $\lambda=0.25,0.5$, and 0.7 .

(1) Ambiguity measures given Table 13 help the buyer how to choose the best discount policy in TPIFQD and TPIIFQD.

(2) In the Ambiguity measure TPIFQD, for the parameter Value 0.25 , the optimal Value is 56.64 and, for 0.5 , the optimal Value is 48.75. And for the parameter Value 0.75 , the optimal Value is 38.1.
TABLE 12: Solution for the Value-based TPIFQD and TPIIFQD.

\begin{tabular}{lcc}
\hline Value & TPIFQD & TPIIFQD \\
\hline$\lambda=0.25$ & 261 & 319.72 \\
$\lambda=0.5$ & 219 & 256.88 \\
$\lambda=0.75$ & 191.7 & 236.98 \\
\hline
\end{tabular}

TABle 13: Solution for the Ambiguity-based TPIFQD and TPIIFQD.

\begin{tabular}{lcc}
\hline Value & TPIFQD & TPIIFQD \\
\hline$\lambda=0.25$ & 56.64 & 55.31 \\
$\lambda=0.5$ & 48.75 & 50.36 \\
$\lambda=0.75$ & 38.1 & 43.05 \\
\hline
\end{tabular}

(3) In the Ambiguity index TPIIFQD, as the parameter Values increased $(\lambda=0.25,0.5$, and 0.75$)$, the optimal Value decreased (55.31, 50.31, and 43.05)

(4) Here, the TPIFQD Value is less than the TPIIFQD Value for $\lambda=0.50$ and 0.75 , but in the case of $\lambda=0.25$, it is vice versa.

\section{Conclusions and Future Work}

In the classical method, all quantity discount scheme is usually less than the incremental quantity discount scheme, but in this research, there is a difference in the trapezoidal all quantity discount scheme. In the Value and Ambiguity index trapezoidal incremental quantity discount scheme, for the parameter Value 0.75 , the all quantity is greater and the incremental quantity is smaller in Numerical Example 1. And, for the parameter Value 0.25 , the all quantity is greater and the incremental quantity is smaller. Hence, the intuitionistic fuzzy transportation model is more practical and flexible in nature. The theory of Value and Ambiguity index discussed here is very extensive and can be applied to other areas of operational research such as the supply chain model and market research. This research proposed that this method is more effective than the classical method. The future direction is to examine the possibilities for the formation of dual results of intuitionistic fuzzy transportation problem with quantity discounts using different heuristic methods. One can extend the concept for solving transportation problems with total quantity discounts and incremental quantity discounts by considering type 2 fuzzy numbers or neutrosophic numbers as parameters. To improve the solution of the intuitionistic fuzzy quantity discount problems, one may use the nonlinear membership and nonmembership functions.

\section{Nomenclature}

IFN: Intuitionistic fuzzy number

IVIFTP: Interval-valued intuitionistic fuzzy transportation problem

IVTIFN: Interval-valued trapezoidal intuitionistic fuzzy number

TrIFN: Trapezoidal intuitionistic fuzzy number 
TPIFQD: Transportation problem with intuitionistic fuzzy quantity discounts

TPIIFQD: Transportation problem with incremental intuitionistic fuzzy quantity discounts.

\section{Data Availability}

No data were used to support the findings of this study.

\section{Conflicts of Interest}

The authors declare that they have no conflicts of interest.

\section{References}

[1] K. Atanassov, Intuitionistic Fuzzy Sets, Theory and Applications, Physica-Verlag, Heidelberg, Germany, 1999.

[2] V. B. Chandran and A. Perry, "Transportations type problems with quantity discounts," Naval Research Logistics Quarterly, vol. 23, no. 2, pp. 195-209, 1976.

[3] C. Das, "An algorithm for selecting quantity discount from realistic schedules," Journal of the Operational Research Society, vol. 41, no. 2, pp. 165-172, 1996.

[4] A. H. I. Lee, H.-Y. Kang, and C.-M. Lai, "Solving lot-sizing problem with quantity discount and transportation cost," International Journal of Systems Science, vol. 44, no. 4, pp. 760-774, 2013.

[5] D. Acharya, M. Basu, and A. Das, "Discounted generalized transportation problem," International Journal of Scientific and Research Publications, vol. 3, no. 7, pp. 1-6, 2013.

[6] I. H. Mubarack Ahmed and A. Emmanuel, "The network transportation problem with volume discount on the shipping cost," International Journal of Science and Research, vol. 3, no. 12, Article ID OCT141439, 2010.

[7] Q. George, O. Chukwadi, and O. Jude, "Transportation algorithm with volume discount on distribution cost (a case study of the Nigerian bottling company plc owerri plant)," American Journal of Applied Mathematics and Statistics, vol. 2, no. 5, pp. 318-323, 2014.

[8] A. Ebrahimnejad and J. L. Verdegay, "A new approach for solving fully intuitionistic fuzzy transportation problems," Fuzzy Optimization and Decision Making, vol. 17, no. 4, pp. $447-474,2018$.

[9] D. K. Jana, "Novel arithmetic operations on type-2 intuitionistic fuzzy and its applications to transportation problem," Pacific Science Review A: Natural Science and Engineering, vol. 18, no. 3, pp. 178-189, 2016.

[10] S. Dinagar and K. Thiripurasundari, "A navel method for solving fuzzy transportation problem involving intuitionistic trapezoidal fuzzy numbers," International Journal of Current Research, vol. 6, no. 6, pp. 7038-7041, 2014.

[11] P. A. Kokila, A. Anju, and B. Radhakrishnan, "Optimality of intuitionistic fuzzy fractional transportation problem of type2," Arab Journal of Basic and Applied Sciences, vol. 26, no. 1, pp. 519-530, 2019.

[12] A. Anju, "Solving hexagonal intuitionistic fuzzy fractional transportation problem using ranking and Russell's method," World Scientific News, vol. 133, pp. 234-247, 2019.

[13] P. V. Lakshmi and J. M. Vinotha, "Multi-objective restricted solid transportation problem in intuitionistic fuzzy environment with emission cost," IJRTE, vol. 8, no. 23, 2019.

[14] A. Mishra, A. Kumar, and M. A. Khan, "A note on "transportation problem under interval-valued intuitionistic fuzzy environment"” Journal of Intelligent \& Fuzzy Systems, vol. 37, no. 1, pp. 897-900, 2019.

[15] M. Darehmiraki, "A novel parametric ranking method for intuitionistic fuzzy numbers," Iranian Journal of Fuzzy Systems, vol. 16, no. 1, pp. 129-143, 2019.

[16] B. Anushya, B. Ramaand, and L. Sudha, "Transportation problem using intuitionistic decagonal fuzzy number," IJRAR, vol. 6, no. 1, pp. 271-277, 2019.

[17] S. A. Edalatpanah, "A data envelopment analysis model with triangular intuitionistic fuzzy numbers," International Journal of Data Envelopment Analysis, vol. 7, no. 4, pp. 47-58, Article ID IJDEA-00422, 2019.

[18] S. A. Edalatpanah and S. Shahabi, "A new two phase method for the fuzzy primal simplex method," International Review of Pure and Applied Mathematics, vol. 8, no. 2, pp. 157-164, 2012.

[19] S. K. Das, T. Mandal, and S. A. Edalatpanah, "A mathematical model for solving fully fuzzy linear programming problem with trapezoidal fuzzy numbers," Applied Intelligence, vol. 46, no. 3, pp. 509-519, 2017.

[20] J. Pratihar, R. Kumar, S. A. Edalatpanah, and A. Dey, "Modified Vogel's approximation method for transportation problem under uncertain environment," Complex \& Intelligent Systems, 2020.

[21] R. Kumar, S. A. Edalatpanah, and S. R. Singh, "A Pythagorean fuzzy approach to the transportation problem," Complex \& Intelligent Systems, vol. 5, pp. 255-263, 2019.

[22] Li and D. Feng, "Decision and game theory in management with intuitionistic fuzzy sets," Studies in Fuzzy and Soft Computing, Vol. 308, Springers, Berlin, Germany, 2014.

[23] S. A. Edalatpanah, "Data envelopment analysis based on triangular neutrosophic numbers," CAAI Transactions on Intelligence Technology, vol. 5, no. 2, pp. 94-98, 2020.

[24] S. A. Edalatpanah, "Neutrosophic structured element," Expert Systems, vol. 37, no. 5, 2020.

[25] A. Bagheri, E. Ebrahimnejad, S. Razavyan, F. H. Lotf, and N. Malekmohammadi, "Fuzzy arithmetic DEA approach for fuzzy multi-objective transportation problem," Operational Research, 2020.

[26] A. Ebrahimnejad, S. Tabatabaei, and F. J. Santos-Arteaga, “A novel lexicographic optimization method for solving shortest path problems with interval-valued triangular fuzzy arc weights," Journal of Intelligent \& Fuzzy Systems, vol. 39, no. 1, pp. 1277-1287, 2020.

[27] A. Ebrahimnejad, "A new method for solving fuzzy transportation problem with $L R$ flat fuzzy numbers," Information Sciences, vol. 357, pp. 108-124, 2016.

[28] A. Ebrahimnejad and J. L. Verdegay, "An efficient computational approach for solving type-2 intuitionistic fuzzy numbers based transportation problems," International Journal of Computational Intelligence Systems, vol. 9, no. 6, pp. 1154-1173, 2016. 